\title{
Semidefinite approximations of projections and polynomial images of semi-algebraic sets
}

\author{
Victor Magron $^{1} \quad$ Didier Henrion ${ }^{2,3,4} \quad$ Jean-Bernard Lasserre ${ }^{2,3}$
}

July 23, 2015

\begin{abstract}
Given a compact semi-algebraic set $\mathbf{S} \subset \mathbb{R}^{n}$ and a polynomial map $f: \mathbb{R}^{n} \rightarrow \mathbb{R}^{m}$, we consider the problem of approximating the image set $\mathbf{F}=f(\mathbf{S}) \subset \mathbb{R}^{m}$. This includes in particular the projection of $\mathbf{S}$ on $\mathbb{R}^{m}$ for $n \geq m$. Assuming that $\mathbf{F} \subset \mathbf{B}$, with $\mathbf{B} \subset \mathbb{R}^{m}$ being a "simple" set (e.g. a box or a ball), we provide two methods to compute certified outer approximations of $\mathbf{F}$. Method 1 exploits the fact that $\mathbf{F}$ can be defined with an existential quantifier, while Method 2 computes approximations of the support of image measures. The two methods output a sequence of superlevel sets defined with a single polynomial that yield explicit outer approximations of $\mathbf{F}$. Finding the coefficients of this polynomial boils down to computing an optimal solution of a convex semidefinite program. We provide guarantees of strong convergence to $\mathbf{F}$ in $L_{1}$ norm on $\mathbf{B}$, when the degree of the polynomial approximation tends to infinity. Several examples of applications are provided, together with numerical experiments.
\end{abstract}

Keywords semi-algebraic sets; semidefinite programming; moment relaxations; polynomial sums of squares.

\section{Introduction}

Consider a polynomial map $f: \mathbb{R}^{n} \rightarrow \mathbb{R}^{m}, \mathbf{x} \mapsto f(\mathbf{x}):=\left(f_{1}(\mathbf{x}), \ldots, f_{m}(\mathbf{x})\right) \in \mathbb{R}^{m}[\mathbf{x}]$ of degree $d:=\max \left\{\operatorname{deg} f_{1}, \ldots, \operatorname{deg} f_{m}\right\}$ and a compact basic semi-algebraic set

$$
\mathbf{S}:=\left\{\mathbf{x} \in \mathbb{R}^{n}: g_{1}^{\mathbf{S}}(\mathbf{x}) \geq 0, \ldots, g_{n}^{\mathbf{S}}(\mathbf{x}) \geq 0\right\}
$$

defined by polynomials $g_{1}^{\mathbf{S}}, \ldots, g_{n}^{\mathbf{S}} \in \mathbb{R}[\mathbf{x}]$.

\footnotetext{
${ }^{1}$ Circuits and Systems Group, Department of Electrical and Electronic Engineering, Imperial College London, South Kensington Campus, London SW7 2AZ, UK.

${ }^{2}$ CNRS; LAAS; 7 avenue du colonel Roche, F-31400 Toulouse; France.

${ }^{3}$ Université de Toulouse; LAAS, F-31400 Toulouse, France.

${ }^{4}$ Faculty of Electrical Engineering, Czech Technical University in Prague, Technická 2, CZ-16626 Prague, Czech Republic
} 
Since $\mathbf{S}$ is compact, the image set

$$
\mathbf{F}:=f(\mathbf{S})
$$

is included in a basic compact semi-algebraic set $\mathbf{B}$, assumed to be "simple" (e.g. a box or a ball) and described by

$$
\mathbf{B}:=\left\{\mathbf{y} \in \mathbb{R}^{m}: g_{1}^{\mathbf{B}}(\mathbf{y}) \geq 0, \ldots, g_{n^{\mathbf{B}}}^{\mathbf{B}}(\mathbf{y}) \geq 0\right\}
$$

for some polynomials $g_{1}^{\mathbf{B}}, \ldots, g_{n^{\mathrm{B}}}^{\mathbf{B}} \in \mathbb{R}[\mathbf{y}]$.

The purpose of this paper is to approximate $\mathbf{F}$, the image of $\mathbf{S}$ under the polynomial map $f$, with superlevel sets of single polynomials of fixed degrees. One expects the approximation to be tractable, i.e. to be able to control the degree of the polynomials used to define the approximations. This appears to be quite a challenging problem since the polynomial map $f$ and the set $\mathbf{S}$ can be both complicated.

This problem includes two important special cases. The first problem is to approximate the projection of $\mathbf{S}$ on $\mathbb{R}^{m}$ for $n \geq m$. The second problem is the approximation of Pareto curves in the context of multicriteria optimization. In [MHL14, we reformulate this second problem through parametric polynomial optimization, which can be solved using a hierarchy of semidefinite approximations. The present work proposes an alternative solution via approximations of polynomial images of semi-algebraic sets.

In the case of semi-algebraic set projections, notice that computer algebra algorithms provide an exact description of the projection. These algorithms are based on real quantifier elimination (see e.g. [Tar51, Col74, BPR96]). For state-of-the-art computer algebra algorithms for quantifier elimination, we refer the interested reader to the survey [Bas14] and the references therein. Quantifier elimination can be performed with the famous cylindrical algebraic decomposition algorithm. For a finite set of $s$ polynomials in $n$ variables, the (time) computational complexity of this algorithm is bounded by $(s d)^{2^{O(n)}}$, thus doubly exponential [Col74, W7̈6]. In [GJ88, an algorithm was proposed to find real elements of semi-algebraic sets in sub-exponential time. The Block Elimination Algorithm is a singly exponential algorithm to eliminate one block of $n$ variables out of $n+m$ variables, with a complexity bounded by $s^{n+1} d^{O(n+m)}$ (see [BPR06. Chapter 14] for the formalization of this algorithm). For applications that satisfy certain additional assumptions (e.g. radicality, equidimensionality, etc.), one can use the variant quantifier elimination method proposed in [HD12, which is less computationally demanding.

Providing approximation algorithms for quantifier elimination is interesting on its own because it may provide simpler answers than exact methods, with a more reasonable computational cost. On the one hand, we do not require an exact description of the projection but rather a hierarchy of outer approximations with a guarantee of convergence to the exact projection. On the other hand, the present methodology only requires the following assumptions: 1) the set $\mathbf{S}$ is compact and 2) either the semi-algebraic set $\mathbf{S}$ or $\mathbf{F}($ resp. $\mathbf{B} \backslash \mathbf{F})$ has nonempty interior.

Contribution and general methodology We provide two methods to approximate the image of semi-algebraic sets under polynomial applications. 
- Method 1 consists of rewriting $\mathbf{F}$ as a set defined with an existential quantifier. Then, one can outer approximate $\mathbf{F}$ as closely as desired with a hierarchy of superlevel sets of the form $\mathbf{F}_{r}^{1}:=\left\{\mathbf{y} \in \mathbf{B}: q_{r}(\mathbf{y}) \geq 0\right\}$ for some polynomials $q_{r} \in \mathbb{R}[\mathbf{y}]$ of increasing degrees $2 r$.

- Method 2 consists of building a hierarchy of relaxations for the infinite dimensional moment problem whose optimal value is the volume of $\mathbf{F}$ and whose optimum is the restriction of the Lebesgue measure on $\mathbf{F}$. Then, one can outer approximate $\mathbf{F}$ as closely as desired with a hierarchy of super level sets of the form $\mathbf{F}_{r}^{2}:=\{\mathbf{y} \in \mathbf{B}$ : $\left.w_{r}(\mathbf{y}) \geq 1\right\}$, for some polynomials $w_{r} \in \mathbb{R}[\mathbf{y}]$ of increasing degrees $2 r$.

Method 1 and Method 2 share the following essential features:

1. The sets $\mathbf{F}_{r}^{1}$ and $\mathbf{F}_{r}^{2}$ are described with a single polynomial of degree $2 r$.

2. Assuming non-emptiness of the interior of $\mathbf{S}$, resp. of $\mathbf{F}$ and $\mathbf{B} \backslash \mathbf{F}$, one has $\lim _{r \rightarrow \infty} \operatorname{vol}\left(\mathbf{F}_{r}^{1} \backslash \mathbf{F}\right)=0$, resp. $\lim _{r \rightarrow \infty} \operatorname{vol}\left(\mathbf{F}_{r}^{2} \backslash \mathbf{F}\right)=0$, where $\operatorname{vol}(\cdot)$ stands for the volume or Lebesgue measure.

3. Computing the coefficient vectors of the polynomials $\left(q_{r}\right)_{r \in \mathbb{N}}$, resp. $\left(w_{r}\right)_{r \in \mathbb{N}}$, boils down to finding optimal solutions of a hierarchy of semidefinite programs. The size of these programs is parametrized by the relaxation order $r$ and depends on the number of variables $n$, the number $m$ of components of the polynomial $f$ as well as its degree $d$. For the hierarchy of semidefinite programs associated with Method 1, the number of variables at step $r$ is bounded by $\left(\begin{array}{c}n+m+2 r \\ 2 r\end{array}\right)$, with $\left(n^{\mathbf{S}}+n^{\mathbf{B}}+1\right)$ semidefinite constraints of size at most $\left(\begin{array}{c}n+m+r \\ r\end{array}\right)$. Step $r$ of the semidefinite hierarchy associated with Method 2 involves at most $\left(\begin{array}{c}n+2 r d \\ 2 r d\end{array}\right)+2\left(\begin{array}{c}m+2 r \\ 2 r\end{array}\right)$ variables, $\left(n_{\mathbf{S}}+1\right)$ semidefinite constraints of size at most $\left(\begin{array}{c}n+r d \\ r d\end{array}\right)$ and $2\left(n^{\mathbf{B}}+1\right)$ semidefinite constraints of size at $\operatorname{most}\left(\begin{array}{c}m+r \\ r\end{array}\right)$.

4. Data sparsity can be exploited to reduce the overall computational cost.

Method 1 relies on the previous study [Las15], in which the author obtains tractable approximations of sets defined with existential quantifiers. The present article provides an extension of the result of [Las15, Theorem 3.4], where one does not require anymore that some set has zero Lebesgue measure. This is mandatory to prove the volume convergence result.

In [HLS09, the authors consider the problem of approximating the volume of a general compact basic semi-algebraic set. The initial problem is then reformulated as an infinite dimensional linear programming (LP) problem, whose unknown is the restriction of the Lebesgue measure on the set of interest. The main idea behind Method 2 is a similar infinite dimensional LP reformulation of the problem, whose unknown is $\mu_{1}$, the restriction of the Lebesgue measure on $\mathbf{F}$. One ends up in computing a finite number of moments of the measure $\mu_{0}$ supported on $\mathbf{S}$ such that the image of $\mu_{0}$ under $f$ is precisely $\mu_{1}$. Note however that there is an important novelty compared with [HLS09, in which the set under study is explicitly described as a basic compact semi-algebraic set (i.e. the intersection of superlevel sets of known polynomials), whereas such a description is not known for $\mathbf{F}$. 
Structure of the paper The paper is organized as follows. Section 2 recalls the basic background about polynomial sum of squares approximations, moment and localizing matrices. Section 3 presents our approximation method for existential quantifier elimination (Method 1). Section 4 is dedicated to the support of image measures (Method 2). In Section 5, we analyze the theoretical complexity of both methods and describe how the system sparsity can be exploited. Section 6 presents several examples where Method 1 and Method 2 are successfully applied.

\section{Notation and Definitions}

Let $\mathbb{R}[\mathbf{x}]$ (resp. $\mathbb{R}_{2 r}[\mathbf{x}]$ ) be the ring of real polynomials (resp. of degree at most $2 r$ ) in the variable $\mathbf{x}=\left(x_{1}, \ldots, x_{n}\right) \in \mathbb{R}^{n}$, for $r \in \mathbb{N}$. With $\mathbf{S}$ a basic semi-algebraic set as in (1), we set $r_{j}^{\mathbf{S}}:=\left\lceil\left(\operatorname{deg} g_{j}^{\mathbf{S}}\right) / 2\right\rceil, j=1, \ldots, n^{\mathbf{S}}$ and with $\mathbf{B}$ a basic semi-algebraic set as in (2), we set $r_{j}^{\mathbf{B}}:=\left\lceil\left(\operatorname{deg} g_{j}^{\mathbf{B}}\right) / 2\right\rceil, j=1, \ldots, n^{\mathbf{B}}$. Let $\Sigma[\mathbf{x}]$ denote the cone of sum of squares (SOS) of polynomial, and let $\Sigma_{r}[\mathbf{x}]$ denote the cone of polynomials SOS of degree at most $2 r$, that is $\Sigma_{r}[\mathbf{x}]:=\Sigma[\mathbf{x}] \cap \mathbb{R}_{2 r}[\mathbf{x}]$.

For the ease of notation, we set $g_{0}^{\mathbf{S}}(\mathbf{x}):=1$ and $g_{0}^{\mathbf{B}}(\mathbf{y}):=1$. For each $r \in \mathbb{N}$, let $\mathbf{Q}_{r}(\mathbf{S})$ (resp. $\mathbf{Q}_{r}(\mathbf{B})$ ) be the $r$-truncated quadratic module (a convex cone) generated by $g_{0}^{\mathbf{S}}, \ldots, g_{n_{\mathbf{S}}}^{\mathbf{S}}\left(\operatorname{resp} . g_{0}^{\mathbf{B}}, \ldots, g_{n_{\mathrm{B}}}^{\mathbf{B}}\right)$ :

$$
\begin{aligned}
\mathbf{Q}_{r}(\mathbf{S}) & :=\left\{\sum_{j=0}^{n^{\mathbf{S}}} s_{j}(\mathbf{x}) g_{j}^{\mathbf{S}}(\mathbf{x}): s_{j} \in \Sigma_{r-r_{j}^{\mathbf{S}}}[\mathbf{x}], j=0, \ldots, n^{\mathbf{S}}\right\}, \\
\mathbf{Q}_{r}(\mathbf{B}) & :=\left\{\sum_{j=0}^{n^{\mathbf{B}}} s_{j}(\mathbf{y}) g_{j}^{\mathbf{B}}(\mathbf{y}): s_{j} \in \Sigma_{r-r_{j}^{\mathbf{B}}}[\mathbf{y}], j=0, \ldots, n^{\mathbf{B}}\right\} .
\end{aligned}
$$

Now, we introduce additional notations which are required for Method 1. Let us first describe the product set

$$
\mathbf{K}:=\mathbf{S} \times \mathbf{B}=\left\{(\mathbf{x}, \mathbf{y}) \in \mathbb{R}^{n+m}: g_{1}(\mathbf{x}, \mathbf{y}) \geq 0, \ldots, g_{n} \mathbf{K}(\mathbf{x}, \mathbf{y}) \geq 0\right\} \subset \mathbb{R}^{n+m},
$$

with $n^{\mathbf{K}}:=n^{\mathbf{S}}+n^{\mathbf{B}}$ and the polynomials $g_{j} \in \mathbb{R}[\mathbf{x}, \mathbf{y}], j=1, \ldots, n^{\mathbf{K}}$ are defined by:

$$
g_{j}(\mathbf{x}, \mathbf{y}):= \begin{cases}g_{j}^{\mathbf{S}}(\mathbf{x}) & \text { if } 1 \leq j \leq n^{\mathbf{S}} \\ g_{j}^{\mathbf{B}}(\mathbf{y}) & \text { if } n^{\mathbf{S}}+1 \leq j \leq n^{\mathbf{K}} .\end{cases}
$$

As previously, we set $r_{j}^{\mathbf{K}}:=\left\lceil\left(\operatorname{deg} g_{j}\right) / 2\right\rceil, j=1, \ldots, n^{\mathbf{K}}, g_{0}(\mathbf{x}, \mathbf{y}):=1$ and $\mathbf{Q}_{r}(\mathbf{K})$ stands for the $r$-truncated quadratic module generated by the polynomials $g_{0}, \ldots, g_{n} \mathrm{~K}$.

To guarantee the theoretical convergence of our two methods, we need to assume the existence of the following algebraic certificates of boundedness of the sets $\mathbf{S}$ and $\mathbf{B}$ :

Assumption 2.1. There exists an integer $j^{\mathbf{S}}\left(\right.$ resp. $j^{\mathbf{B}}$ ) such that $g_{j}^{\mathbf{S}}=g^{\mathbf{S}}:=N^{\mathbf{S}}-\|\mathbf{x}\|_{2}^{2}$ (resp. $\left.g_{j^{\mathrm{B}}}^{\mathbf{B}}=g^{\mathbf{B}}:=N^{\mathbf{B}}-\|\mathbf{y}\|_{2}^{2}\right)$ for large enough positive integers $N^{\mathbf{S}}$ and $N^{\mathbf{B}}$. 
For every $\alpha \in \mathbb{N}^{n}$ the notation $\mathbf{x}^{\alpha}$ stands for the monomial $x_{1}^{\alpha_{1}} \ldots x_{n}^{\alpha_{n}}$ and for every $r \in \mathbb{N}$, let $\mathbb{N}_{r}^{n}:=\left\{\alpha \in \mathbb{N}^{n}: \sum_{j=1}^{n} \alpha_{j} \leq r\right\}$, whose cardinality is $\left(\begin{array}{c}n+r \\ r\end{array}\right)$. One writes a polynomial $p \in \mathbb{R}[\mathbf{x}, \mathbf{y}]$ as follows:

$$
(\mathbf{x}, \mathbf{y}) \mapsto p(\mathbf{x}, \mathbf{y})=\sum_{(\alpha, \beta) \in \mathbb{N}^{n+m}} p_{\alpha \beta} \mathbf{x}^{\alpha} \mathbf{y}^{\beta}
$$

and we identify $p$ with its vector of coefficients $\mathbf{p}=\left(p_{\alpha \beta}\right)$ in the canonical basis $\left(\mathbf{x}^{\alpha} \mathbf{y}^{\beta}\right)$, $\alpha \in \mathbb{N}^{n}, \beta \in \mathbb{N}^{m}$.

Given a real sequence $\mathbf{z}=\left(z_{\alpha \beta}\right)$, we define the multivariate linear functional $\ell_{\mathbf{z}}: \mathbb{R}[\mathbf{x}, \mathbf{y}] \rightarrow$ $\mathbb{R}$ by $\ell_{\mathbf{z}}(p):=\sum_{\alpha \beta} p_{\alpha \beta} z_{\alpha \beta}$, for all $p \in \mathbb{R}[\mathbf{x}, \mathbf{y}]$.

Moment matrix The moment matrix associated with a sequence $\mathbf{z}=\left(z_{\alpha \beta}\right)_{(\alpha, \beta) \in \mathbb{N}^{n+m}}$, is the real symmetric matrix $\mathbf{M}_{r}(\mathbf{z})$ with rows and columns indexed by $\mathbb{N}_{r}^{n+m}$, and whose entries are defined by:

$$
\mathbf{M}_{r}(\mathbf{z})((\alpha, \beta),(\delta, \gamma)):=\ell_{\mathbf{z}}\left(\mathbf{x}^{\alpha+\delta} \mathbf{y}^{\beta+\gamma}\right), \quad \forall \alpha, \delta \in \mathbb{N}_{r}^{n}, \quad \forall \beta, \gamma \in \mathbb{N}_{r}^{m} .
$$

Localizing matrix The localizing matrix associated with a sequence $\mathbf{z}=\left(z_{\alpha \beta}\right)_{(\alpha, \beta) \in \mathbb{N}^{n+m}}$ and a polynomial $q \in \mathbb{R}[\mathbf{x}, \mathbf{y}]$ (with $q(\mathbf{x}, \mathbf{y})=\sum_{u, v} q_{u v} \mathbf{x}^{u} \mathbf{y}^{v}$ ) is the real symmetric matrix $\mathbf{M}_{r}(q \mathbf{z})$ with rows and columns indexed by $\mathbb{N}_{r}^{n+m}$, and whose entries are defined by:

$$
\mathbf{M}_{r}(q \mathbf{z})((\alpha, \beta),(\delta, \gamma)):=\ell_{\mathbf{z}}\left(q(\mathbf{x}, \mathbf{y}) \mathbf{x}^{\alpha+\delta} \mathbf{y}^{\beta+\gamma}\right), \quad \forall \alpha, \delta \in \mathbb{N}_{r}^{n}, \quad \forall \beta, \gamma \in \mathbb{N}_{r}^{m} .
$$

We define the restriction of the Lebesgue measure on a subset $\mathbf{A} \subset \mathbf{B}$ by $\lambda_{\mathbf{A}}(d \mathbf{y}):=$ $\mathbf{1}_{\mathbf{A}}(\mathbf{y}) d \mathbf{y}$, with $\mathbf{1}_{\mathbf{A}}: \mathbf{B} \rightarrow\{0,1\}$ denoting the indicator function on $\mathbf{A}:$

$$
\mathbf{1}_{\mathbf{A}}(\mathbf{y}):= \begin{cases}1 & \text { if } \mathbf{y} \in \mathbf{A} \\ 0 & \text { otherwise }\end{cases}
$$

The moments of the Lebesgue measure on $\mathbf{B}$ are denoted by

$$
z_{\beta}^{\mathbf{B}}:=\int \mathbf{y}^{\beta} \lambda_{\mathbf{B}}(d \mathbf{y}) \in \mathbb{R}, \quad \beta \in \mathbb{N}^{m}
$$

We assume that the bounding set $\mathbf{B}$ is "simple" in the following sense:

Assumption 2.2. The moments (4) of the Lebesgue measure on $\mathbf{B}$ can be explicitly computed using cubature formula for integration.

\section{Method 1: existential quantifier elimination}

\subsection{Semi-algebraic sets defined with existential quantifiers}

The set $\mathbf{F}=f(\mathbf{S})$ is the image of the compact semi-algebraic set $\mathbf{S}$ under the polynomial map $f: \mathbf{S} \rightarrow \mathbf{B}$, thus it can be defined with an existential quantifier:

$$
\mathbf{F}=\left\{\mathbf{y} \in \mathbf{B}: \exists \mathbf{x} \in \mathbf{S} \text { s.t. } h_{f}(\mathbf{x}, \mathbf{y}) \geq 0\right\}
$$


with

$$
h_{f}: \mathbb{R}^{n+m} \rightarrow \mathbb{R}, \quad(\mathbf{x}, \mathbf{y}) \mapsto h_{f}(\mathbf{x}, \mathbf{y}):=-\|\mathbf{y}-f(\mathbf{x})\|_{2}^{2}=-\sum_{j=1}^{m}\left(y_{j}-f_{j}(\mathbf{x})\right)^{2}
$$

Let us also define

$$
h: \mathbb{R}^{m} \rightarrow \mathbb{R}, \quad \mathbf{y} \mapsto h(\mathbf{y}):=\sup _{\mathbf{x} \in \mathbf{S}} h_{f}(\mathbf{x}, \mathbf{y})
$$

Theorem 3.1. There exists a sequence of polynomials $\left(p_{r}\right)_{r \in \mathbb{N}} \subset \mathbb{R}[\mathbf{y}]$ such that $p_{r}(\mathbf{y}) \geq$ $h_{f}(\mathbf{x}, \mathbf{y})$ for all $r \in \mathbb{N}, \mathbf{x} \in \mathbf{S}, \mathbf{y} \in \mathbf{B}$ and such that

$$
\lim _{r \rightarrow \infty} \int\left|p_{r}(\mathbf{y})-h(\mathbf{y})\right| \lambda_{\mathbf{B}}(d \mathbf{y})=0 .
$$

Proof. The result follows readily from [Las15, Theorem 3.1 (3.4)] with the notations $\mathbf{x} \leftarrow \mathbf{y}, \mathbf{y} \leftarrow \mathbf{x}, \mathbf{K} \leftarrow \mathbf{B} \times \mathbf{S}, \mathbf{K}_{\mathbf{x}} \leftarrow \mathbf{S} \neq \emptyset$ and $J_{f} \leftarrow-h$, which is lower semicontinuous.

Theorem 3.2. For each $r \in \mathbb{N}$, define $\mathbf{F}_{r}:=\left\{\mathbf{y} \in \mathbf{B}: p_{r}(\mathbf{y}) \geq 0\right\}$ where the sequence of polynomials $\left(p_{r}\right)_{r \in \mathbb{N}} \subset \mathbb{R}[\mathbf{y}]$ is as in Theorem 3.1. Then $\mathbf{F}_{r} \supset \mathbf{F}$ and one has

$$
\lim _{r \rightarrow \infty} \operatorname{vol}\left(\mathbf{F}_{r} \backslash \mathbf{F}\right)=0 .
$$

Proof. Let $r \in \mathbb{N}$. By assumption, one has $p_{r}(\mathbf{y}) \geq h_{f}(\mathbf{x}, \mathbf{y})$ for all $\mathbf{x} \in \mathbf{S}, \mathbf{y} \in \mathbf{B}$. Thus, one has $p_{r}(\mathbf{y}) \geq h(\mathbf{y})$ for all $\mathbf{y} \in \mathbf{B}$, which implies that $\mathbf{F}_{r} \supset \mathbf{F}$.

It remains to prove (6). Let us define $\mathbf{F}(k):=\{\mathbf{y} \in \mathbf{B}: h(\mathbf{y}) \geq-1 / k\}$. First, we show that

$$
\lim _{k \rightarrow \infty} \operatorname{vol} \mathbf{F}(k)=\operatorname{vol} \mathbf{F} .
$$

For each $k \in \mathbb{N}$, one has $\mathbf{F}(k+1) \subseteq \mathbf{F}(k) \subseteq \mathbf{B}$, thus the sequence of indicator functions $\left(\mathbf{1}_{\mathbf{F}(k)}\right)_{k \in \mathbb{N}}$ is non-increasing and bounded. Next, let us show that for all $\mathbf{y} \in \mathbf{B}, \mathbf{1}_{\mathbf{F}(k)}(\mathbf{y}) \rightarrow$ $\mathbf{1}_{\mathbf{F}}(\mathbf{y})$, as $k \rightarrow \infty$ :

- Let $\mathbf{y} \in \mathbf{F}$. By the inclusion $\mathbf{F} \subseteq \mathbf{F}(k)$, for each $k \in \mathbb{N}, \mathbf{1}_{\mathbf{F}(k)}(\mathbf{y})=\mathbf{1}_{\mathbf{F}}(\mathbf{y})=1$ and the result trivially holds.

- Let $\mathbf{y} \in \mathbf{B} \backslash \mathbf{F}$, so there exists $\epsilon>0$ such that $h(\mathbf{y})=-\epsilon$. Thus, there exists $k_{0} \in \mathbb{N}$ such that for all $k \geq k_{0}, \mathbf{y} \in \mathbf{B} \backslash \mathbf{F}(k)$.

Hence, $\mathbf{1}_{\mathbf{F}(k)}(\mathbf{y}) \rightarrow \mathbf{1}_{\mathbf{F}}(\mathbf{y})$ for each $\mathbf{y} \in \mathbf{B}$, as $k \rightarrow \infty$ (monotone non-increasing). By the Monotone Convergence Theorem, $\mathbf{1}_{\mathbf{F}(k)}(\mathbf{y}) \rightarrow \mathbf{1}_{\mathbf{F}}(\mathbf{y})$ for the $L_{1}$ norm on $\mathbf{B}$ and (7) holds. Next, we prove that for each $k \in \mathbb{N}$,

$$
\lim _{r \rightarrow \infty} \operatorname{vol} \mathbf{F}_{r} \leq \operatorname{vol} \mathbf{F}(k)
$$


By Theorem 3.1 applied to the sequence $\left(p_{r}\right)_{r \in \mathbb{N}}$, one has $\lim _{r \rightarrow \infty} \int\left|p_{r}(\mathbf{y})-h(\mathbf{y})\right| \lambda_{\mathbf{B}}(d \mathbf{y})=$ 0 . Thus, by [Ash72, Theorem 2.5.1], the sequence $\left(p_{r}\right)_{r \in \mathbb{N}}$ converges to $h$ in measure, i.e. for every $\epsilon>0$,

$$
\lim _{r \rightarrow \infty} \operatorname{vol}\left(\left\{\mathbf{y} \in \mathbf{B}:\left|p_{r}(\mathbf{y})-h(\mathbf{y})\right| \geq \epsilon\right\}\right)=0 .
$$

For every $k \geq 1$, observe that:

$$
\begin{aligned}
\operatorname{vol} \mathbf{F}_{r}= & \operatorname{vol}\left(\mathbf{F}_{r} \cap\left\{\mathbf{y} \in \mathbf{B}:\left|p_{r}(\mathbf{y})-h(\mathbf{y})\right| \geq 1 / k\right\}\right)+ \\
& \operatorname{vol}\left(\mathbf{F}_{r} \cap\left\{\mathbf{y} \in \mathbf{B}:\left|p_{r}(\mathbf{y})-h(\mathbf{y})\right|<1 / k\right\}\right) .
\end{aligned}
$$

It follows from (9) that $\lim _{r \rightarrow \infty} \operatorname{vol}\left(\mathbf{F}_{r} \cap\left\{\mathbf{y} \in \mathbf{B}:\left|p_{r}(\mathbf{y})-h(\mathbf{y})\right| \geq 1 / k\right\}\right)=0$. In addition, for all $r \in \mathbb{N}$,

$$
\operatorname{vol}\left(\mathbf{F}_{r} \cap\left\{\mathbf{y} \in \mathbf{B}:\left|p_{r}(\mathbf{y})-h(\mathbf{y})\right|<1 / k\right\}\right) \leq \operatorname{vol}(\{\mathbf{y} \in \mathbf{B}: h(\mathbf{y}) \geq-1 / k\})=\operatorname{vol} \mathbf{F}(k) .
$$

Using both (10) and (11), and letting $r \rightarrow \infty$, yields (8). Thus, we have the following inequalities:

$$
\operatorname{vol} \mathbf{F} \leq \lim _{r \rightarrow \infty} \operatorname{vol} \mathbf{F}_{r} \leq \operatorname{vol} \mathbf{F}(k) .
$$

Using (7) and letting $k \rightarrow \infty$ yields the desired result.

\subsection{Practical computation using semidefinite programming}

In this section we show how the sequence of polynomials of Theorems 3.1 and 3.2 can be computed in practice. Define $r_{\min }^{(1)}:=\max \left\{d, r_{1}, \ldots, r_{n} \mathrm{~K}\right\}$. For $r \geq r_{\min }^{(T)}$, consider the following hierarchy of semidefinite programs:

$$
\begin{aligned}
p_{r}^{*}:=\inf _{q} & \sum_{\beta \in \mathbb{N}_{2 r}^{m}} q_{\beta} z_{\beta}^{\mathbf{B}} \\
\text { s.t. } & q-h_{f} \in \mathbf{Q}_{r}(\mathbf{K}), \\
& q \in \mathbb{R}_{2 r}[\mathbf{y}] .
\end{aligned}
$$

The semidefinite program dual of $(12)$ reads:

$$
\begin{aligned}
d_{r}^{*}:=\sup _{\mathbf{z}} & \ell_{\mathbf{z}}\left(h_{f}\right) \\
\text { s.t. } & \mathbf{M}_{r}(\mathbf{z}) \succeq 0, \\
& \mathbf{M}_{r-r_{j}^{\mathbf{K}}}\left(g_{j} \mathbf{z}\right) \succeq 0, \quad j=1, \ldots, n^{\mathbf{K}}, \\
& \ell_{\mathbf{z}}\left(\mathbf{y}^{\beta}\right)=z_{\beta}^{\mathbf{B}}, \quad \forall \beta \in \mathbb{N}_{2 r}^{m} .
\end{aligned}
$$

Theorem 3.3. Let $r \geq r_{\min }^{(1)}$ and suppose that Assumption 2.1 holds. Then:

1. $p_{r}^{*}=d_{r}^{*}$, i.e. there is no duality gap between the semidefinite program (12) and its dual (13). 
2. The semidefinite program (13) has an optimal solution. In addition, if $\mathbf{S}$ has nonempty interior, then the semidefinite program (12) has an optimal solution $q_{r}$, and the sequence $\left(q_{r}\right)_{r \in \mathbb{N}}$ converges to $h$ in $L_{1}$ norm on $\mathbf{B}$ :

$$
\lim _{r \rightarrow \infty} \int\left|q_{r}(\mathbf{y})-h(\mathbf{y})\right| \lambda_{\mathbf{B}}(d \mathbf{y})=0 .
$$

3. Defining the set

$$
\mathbf{F}_{r}^{1}:=\left\{\mathbf{y} \in \mathbf{B}: q_{r}(\mathbf{y}) \geq 0\right\}
$$

it holds that

$$
\mathbf{F}_{r}^{1} \supset \mathbf{F}
$$

and

$$
\lim _{r \rightarrow \infty} \operatorname{vol}\left(\mathbf{F}_{r}^{1} \backslash \mathbf{F}\right)=0 .
$$

Proof. 1. Let $\mathcal{D}_{r}$ (resp. $\left.\mathcal{D}_{r}^{*}\right)$ stand for the feasible (resp. optimal) solution set of the semidefinite program (13). First, we prove that $\mathcal{D}_{r} \neq \emptyset$. Let $\mathbf{z}=\left(z_{\alpha \beta}\right)_{(\alpha, \beta) \in \mathbb{N}^{n+m}}$ be the sequence moments of $\lambda_{\mathbf{K}}$, the Lebesgue measure on $\mathbf{K}=\mathbf{S} \times \mathbf{B}$. Since the measure is supported on $\mathbf{K}$, the semidefinite constraints $\mathbf{M}_{r}(\mathbf{z}) \succeq 0, \mathbf{M}_{r-r_{j}^{\mathbf{K}}}\left(g_{j} \mathbf{z}\right) \succeq$ $0, j=1, \ldots, n^{\mathbf{K}}$ are satisfied. By construction, the marginal of $\lambda_{\mathbf{K}}$ on $\mathbf{B}$ is $\lambda_{\mathbf{B}}$ and the following equality constraints are satisfied: $\ell_{\mathbf{z}}\left(\mathbf{y}^{\beta}\right)=z_{\beta}^{\mathbf{B}}$ for all $\beta \in \mathbb{N}_{2 r}^{m}$. Thus, the finite sequence $\mathbf{z}$ lies in $\mathcal{D}_{r} \neq \emptyset$.

Note that Assumption 2.1 implies that the semidefinite constraints $\mathbf{M}_{r-1}\left(g^{\mathbf{S}} \mathbf{z}\right) \succeq 0$ and $\mathbf{M}_{r-1}\left(g^{\mathbf{B}} \mathbf{z}\right) \succeq 0$ both hold. Thus, the first diagonal elements of $\mathbf{M}_{r-1}\left(g^{\mathbf{S}} \mathbf{z}\right)$ and $\mathbf{M}_{r-1}\left(g^{\mathbf{B}} \mathbf{z}\right)$ are nonnegative, and since $\ell_{\mathbf{z}}(1)=z_{0}^{\mathbf{B}}$, it follows that $\ell_{\mathbf{z}}\left(x_{i}^{2 k}\right) \leq\left(N^{\mathbf{S}}\right)^{k} z_{0}^{\mathbf{B}}$, $i=1, \ldots, n$ and $\ell_{\mathbf{z}}\left(y_{j}^{2 k}\right) \leq\left(N^{\mathbf{B}}\right)^{k} z_{0}^{\mathbf{B}}, j=1, \ldots, m, k=0, \ldots, r$ and we deduce from [LN07, Lemma 4.3, p. 111] that $\left|z_{\alpha \beta}\right|$ is bounded for all $(\alpha, \beta) \in \mathbb{N}_{2 r}^{n+m}$. Thus, the feasible set $\mathcal{D}_{r}$ is compact as closed and bounded. Hence, the set $\mathcal{D}_{r}^{*}$ is nonempty and bounded. The claim then follows from the sufficient condition of strong duality in $\operatorname{Trn} 05]$.

2. Assume that $\mathbf{S}$ has nonempty interior, so $\mathbf{K}=\mathbf{S} \times \mathbf{B}$ has also nonempty interior. Thus, the feasible solution $\mathbf{z}$ (defined above) satisfies $\mathbf{M}_{r}(\mathbf{z}) \succ 0, \mathbf{M}_{r-r_{j}^{\mathbf{K}}}\left(g_{j} \mathbf{z}\right) \succ 0$, $j=1, \ldots, n^{\mathbf{K}}$, which implies that Slater's condition holds for $(13)$. Note also that the semidefinite program $(12)$ has the trivial feasible solution $q=0$ since $-h_{f}$ is SOS by construction. As a consequence of a now standard result of duality in semidefinite programming (see e.g. [VB94]), the semidefinite program (12) has an optimal solution $q_{r} \in \mathbb{R}_{2 r}[\mathbf{y}]$.

Let us consider a sequence of polynomials $\left(p_{k}\right)_{k \in \mathbb{N}} \subset \mathbb{R}[\mathbf{y}]$ as in Theorem 3.1. Now, fix $\epsilon>0$. By Theorem 3.1, there exists $k_{0} \in \mathbb{N}$ such that

$$
\int\left|p_{k}(\mathbf{y})-h(\mathbf{y})\right| \lambda_{\mathbf{B}}(d \mathbf{y}) \leq \epsilon / 2,
$$

for all $k \geq k_{0}$. Then, observe that the polynomial $p_{k}^{\epsilon}:=p_{k}+\epsilon /(2 \mathrm{vol} \mathbf{B})$ satisfies $p_{k}^{\epsilon}(\mathbf{y})-h_{f}(\mathbf{x}, \mathbf{y})>0$, for all $\mathbf{x} \in \mathbf{S}, \mathbf{y} \in \mathbf{B}$. For $r \in \mathbb{N}$ large enough, as a consequence 
of Putinar's Positivstellensatz (e.g. [Las09, Section 2.5]), there exist $s_{0}, \ldots, s_{n} \mathrm{~K} \in$ $\Sigma[\mathbf{x}, \mathbf{y}]$ such that

$$
p_{k}^{\epsilon}(\mathbf{y})-h_{f}(\mathbf{x}, \mathbf{y})=\sum_{j=0}^{n^{\mathbf{K}}} s_{j}(\mathbf{x}, \mathbf{y}) g_{j}(\mathbf{x}, \mathbf{y}),
$$

with $\operatorname{deg}\left(s_{j} g_{j}\right) \leq 2 r$ for $j=0, \ldots, n^{\mathbf{K}}$. And so, $p_{k}^{\epsilon}-h_{f}$ lies in the $r$-truncated quadratic module $\mathbf{Q}_{r}(\mathbf{K})$, which implies that $p_{k}^{\epsilon}$ is a feasible solution for Problem (12). Hence, $q_{r}$ being an optimal solution of problem (12), the following holds:

$$
\int q_{r}(\mathbf{y}) \lambda_{\mathbf{B}}(d \mathbf{y}) \leq \int p_{k}^{\epsilon}(\mathbf{y}) \lambda_{\mathbf{B}}(d \mathbf{y})=\int\left[p_{k}(\mathbf{y})+\epsilon /(2 \operatorname{vol}(\mathbf{B}))\right] \lambda(d \mathbf{y})
$$

Combining (15) and (16) yields $\int\left|q_{r}(\mathbf{y})-h(\mathbf{y})\right| \lambda(d \mathbf{y}) \leq \epsilon$, concludes the proof.

3. This is a consequence of Theorem 3.2 .

\section{Method 2: support of image measures}

Given a compact set $\mathbf{A} \subset \mathbb{R}^{n}$, let $\mathcal{M}(\mathbf{A})$ stand for the vector space of finite signed Borel measures supported on $\mathbf{A}$, understood as functions from the Borel sigma algebra $\mathcal{B}(\mathbf{A})$ to the real numbers. Let $\mathcal{C}(\mathbf{A})$ stand for the space of continuous functions on $\mathbf{A}$, equipped with the sup-norm (a Banach space). Since $\mathbf{A}$ is compact, the topological dual (i.e. the set of continuous linear functionals) of $\mathcal{C}(\mathbf{A})$ (equipped with the sup-norm), denoted by $\mathcal{C}(\mathbf{A})^{\prime}$, is (isometrically isomorphically identified with) $\mathcal{M}(\mathbf{A})$ equipped with the total variation norm, denoted by $\|\cdot\|_{\mathrm{TV}}$. The cone of non-negative elements of $\mathcal{C}(\mathbf{A})$, resp. $\mathcal{M}(\mathbf{A})$, is denoted by $\mathcal{C}_{+}(\mathbf{A})$, resp. $\mathcal{M}_{+}(\mathbf{A})$. The topology in $\mathcal{C}_{+}(\mathbf{A})$ is the strong topology of uniform convergence while the topology in $\mathcal{M}_{+}(\mathbf{A})$ is the weak-star topology (see [Bar02, Chapter IV] or [Lue97, Section 5.10] for more background on weak-star topology).

Recall that $\lambda_{\mathbf{B}}$ stand for the Lebesgue measure on B. If $\mu, \nu \in \mathcal{M}(\mathbf{A})$, the notation $\mu \ll \nu$ stands for $\mu$ being absolutely continuous w.r.t. $\nu$, whereas the notation $\mu \leq \nu$ means that $\nu-\mu \in \mathcal{M}_{+}(\mathbf{A})$. For background on functional analysis and measure spaces see e.g. [RF10, Section 21.5].

\subsection{LP primal-dual conic formulation}

Given a polynomial application $f: \mathbf{S} \rightarrow \mathbf{B}$, the pushforward or image map

$$
f_{\#}: \mathcal{M}(\mathbf{S}) \rightarrow \mathcal{M}(\mathbf{B})
$$

is defined such that

$$
f_{\#} \mu_{0}(\mathbf{A}):=\mu_{0}(\{\mathbf{x} \in \mathbf{S}: f(\mathbf{x}) \in \mathbf{A}\})
$$

for every set $\mathbf{A} \in \mathcal{B}(\mathbf{B})$ and every measure $\mu_{0} \in \mathcal{M}(\mathbf{S})$. The measure $f_{\#} \mu_{0} \in \mathcal{M}(\mathbf{B})$ is then called the image measure of $\mu_{0}$ under $f$, see e.g. [AFP00, Section 1.5]. 
To approximate the image set $\mathbf{F}=f(\mathbf{S})$, one considers the infinite-dimensional linear programming (LP) problem:

$$
\begin{aligned}
p^{*}:=\sup _{\mu_{0}, \mu_{1}, \hat{\mu}_{1}} & \int \mu_{1} \\
\text { s.t. } & \mu_{1}+\hat{\mu}_{1}=\lambda_{\mathbf{B}}, \\
& \mu_{1}=f_{\#} \mu_{0}, \\
& \mu_{0} \in \mathcal{M}_{+}(\mathbf{S}), \quad \mu_{1}, \hat{\mu}_{1} \in \mathcal{M}_{+}(\mathbf{B}),
\end{aligned}
$$

In the above LP, by definition of the image measure, $\mu_{1}$ exists whenever $\mu_{0}$ is given. The following result gives conditions for $\mu_{0}$ to exist whenever $\mu_{1}$ is given.

Lemma 4.1. Given a measure $\mu_{1} \in \mathcal{M}_{+}(\mathbf{B})$, there is a measure $\mu_{0} \in \mathcal{M}_{+}(\mathbf{S})$ such that $f_{\#} \mu_{0}=\mu_{1}$ if and only if there is no continuous function $v \in \mathcal{C}(\mathbf{B})$ such that $v(f(\mathbf{x})) \geq 0$ for all $\mathbf{x} \in \mathbf{S}$ and $\int v(\mathbf{y}) d \mu_{1}(\mathbf{y})<0$.

Proof. This follows from [CK77, Theorem 6] which is an extension to locally convex topological spaces of the celebrated Farkas Lemma in finite-dimensional linear optimization. One has just to verify that the image cone $f_{\#}\left(\mathcal{M}_{+}(\mathbf{S})\right)=\left\{f_{\#} \mu_{0}: \mu_{0} \in \mathcal{M}_{+}(\mathbf{S})\right\}$ is closed in the weak-star topology $\sigma(\mathcal{M}(\mathbf{B}), \mathcal{C}(\mathbf{B}))$ of $\mathcal{M}(\mathbf{B})$.This, in turn, follows from continuity of $f$ and compactness of $\mathbf{S}$.

Lemma 4.2. LP (17) admits an optimal solution $\left(\mu_{0}^{*}, \mu_{1}^{*}, \hat{\mu}_{1}^{*}\right)$. Moreover, $\mu_{1}^{*}=\lambda_{\mathbf{F}}$ and the optimal value of LP (17) is $p^{*}=\operatorname{vol} \mathbf{F}$.

Proof. First, we prove that for $\mu_{1}^{*}=\lambda_{\mathbf{F}} \in \mathcal{M}_{+}(\mathbf{B})$, there is a measure $\mu_{0}^{*} \in \mathcal{M}_{+}(\mathbf{S})$ such that $f_{\#} \mu_{0}^{*}=\mu_{1}^{*}$. Indeed, by Radon-Nikodým there exists a function $q_{1} \in L_{1}\left(\lambda_{\mathbf{F}}\right)$ such that $d \mu_{1}^{*}(\mathbf{y})=q_{1}(\mathbf{y}) d \lambda_{\mathbf{F}}(\mathbf{y})$ and $q_{1}(\mathbf{y}) \geq 0$ for all $\mathbf{y} \in \mathbf{F}$. The claim follows then from Lemma 4.1 since it is impossible to find a function $v \in \mathcal{C}(\mathbf{B})$ such that $v(\mathbf{y}) \geq 0$ for all $\mathbf{y} \in \mathbf{F}$ while satisfying $\int v(\mathbf{y}) q_{1}(\mathbf{y}) d \lambda_{\mathbf{F}}(\mathbf{y})<0$. Define $\hat{\mu}_{1}^{*}=\lambda_{\mathbf{B}}-\mu_{1}^{*}$, then $\left(\mu_{0}^{*}, \mu_{1}^{*}, \hat{\mu}_{1}^{*}\right)$ is admissible for LP (17). Exactly as in the proof of [HLS09, Theorem 3.1], one finally shows that this triplet is optimum as well as uniqueness of $\mu_{1}^{*}$, yielding the optimal value $p^{*}=\operatorname{vol} \mathbf{F}$.

Next, we express problem (17) as an infinite-dimensional conic problem on appropriate vector spaces. By construction, a feasible solution of problem (17) satisfies:

$$
\begin{aligned}
\int_{\mathbf{B}} v(\mathbf{y}) \mu_{1}(d \mathbf{y})-\int_{\mathbf{S}} v(f(\mathbf{x})) \mu_{0}(d \mathbf{x}) & =0, \\
\int_{\mathbf{B}} w(\mathbf{y}) \mu_{1}(d \mathbf{y})+\int_{\mathbf{B}} w(\mathbf{y}) \hat{\mu}_{1}(d \mathbf{y}) & =\int_{\mathbf{B}} w(\mathbf{y}) \lambda(d \mathbf{y}),
\end{aligned}
$$

for all continuous test functions $v, w \in \mathcal{C}(\mathbf{B})$.

Then, we cast problem (17) as a particular instance of a primal LP in the canonical form given in [Bar02, 7.1.1]:

$$
\begin{aligned}
p^{*}=\sup _{x} & \langle x, c\rangle_{1} \\
\text { s.t. } & \mathcal{A} x=b, \\
& x \in E_{1}^{+},
\end{aligned}
$$

with 
- the vector space $E_{1}:=\mathcal{M}(\mathbf{S}) \times \mathcal{M}(\mathbf{B})^{2}$;

- the vector space $F_{1}:=\mathcal{C}(\mathbf{S}) \times \mathcal{C}(\mathbf{B})^{2}$;

- the duality $\langle\cdot, \cdot\rangle_{1}: E_{1} \times F_{1} \rightarrow \mathbb{R}$, given by the integration of continuous functions against Borel measures, since $E_{1}=F_{1}^{\prime}$;

- the decision variable $x:=\left(\mu_{0}, \mu_{1}, \hat{\mu}_{1}\right) \in E_{1}$ and the reward $c:=(0,1,0) \in F_{1}$;

- $E_{2}:=\mathcal{M}(\mathbf{B})^{2}, F_{2}:=\mathcal{C}(\mathbf{B})^{2}$ and the right hand side vector $b:=(0, \lambda) \in E_{2}=F_{2}^{\prime}$;

- the linear operator $\mathcal{A}: E_{1} \rightarrow E_{2}$ given by

$$
\mathcal{A}\left(\mu_{0}, \mu_{1}, \hat{\mu}_{1}\right):=\left[\begin{array}{c}
-f_{\#} \mu_{0}+\mu_{1} \\
\mu_{1}+\hat{\mu}_{1}
\end{array}\right] .
$$

Notice that all spaces $E_{1}, E_{2}$ (resp. $F_{1}, F_{2}$ ) are equipped with the weak topologies $\sigma\left(E_{1}, F_{1}\right), \sigma\left(E_{2}, F_{2}\right)$ (resp. $\sigma\left(F_{1}, E_{1}\right), \sigma\left(F_{2}, E_{2}\right)$ ). Importantly, $\sigma\left(E_{1}, F_{1}\right)$ is the weak-star topology (since $E_{1}=F_{1}^{\prime}$ ). Observe that $\mathcal{A}$ is continuous with respect to the weak topology, as $\mathcal{A}^{\prime}\left(F_{2}\right) \subset F_{1}$.

With these notations, the dual LP in the canonical form given in [Bar02, 7.1.2] reads:

$$
\begin{aligned}
d^{*}=\inf _{y} & \langle b, y\rangle_{2} \\
\text { s.t. } & \mathcal{A}^{\prime} y-c \in \mathcal{C}_{+}(\mathbf{B})^{2}
\end{aligned}
$$

with

- the dual variable $y:=(v, w) \in E_{2}$;

- the (pre)-dual cone $\mathcal{C}_{+}(\mathbf{B})^{2}$, whose dual is $E_{1}^{+}$;

- the duality pairing $\langle\cdot, \cdot\rangle_{2}: E_{2} \times F_{2} \rightarrow \mathbb{R}$, with $E_{2}=F_{2}^{\prime}$;

- the adjoint linear operator $\mathcal{A}^{\prime}: F_{2} \rightarrow F_{1}$ given by

$$
\mathcal{A}^{\prime}(v, w):=\left[\begin{array}{c}
-v \circ f \\
v+w \\
w
\end{array}\right] .
$$

Using our original notations, the dual LP of problem (17) then reads:

$$
\begin{aligned}
d^{*}:=\inf _{v, w} & \int w(\mathbf{y}) \lambda_{\mathbf{B}}(d \mathbf{y}) \\
\text { s.t. } \quad & v(f(\mathbf{x})) \geq 0, \quad \forall \mathbf{x} \in \mathbf{S}, \\
& w(\mathbf{y}) \geq 1+v(\mathbf{y}), \quad \forall \mathbf{y} \in \mathbf{B}, \\
& w(\mathbf{y}) \geq 0, \quad \forall \mathbf{y} \in \mathbf{B}, \\
& v, w \in \mathcal{C}(\mathbf{B}) .
\end{aligned}
$$


Theorem 4.3. There is no duality gap between problem (17) and problem (22), i.e. $p^{*}=$ $d^{*}$.

Proof. This theorem follows from the "zero duality gap" result from Bar02, Theorem $7.2]$, if one can prove that the cone

$$
\mathcal{A}\left(E_{1}^{+}\right):=\left\{\left(\mathcal{A} x,\langle x, c\rangle_{1}\right): x \in E_{1}^{+}\right\}
$$

is closed in $E_{2} \times \mathbb{R}$. To do so, let us consider a sequence $\left.\left(x^{(k)}\right)=\left(\mu_{0}^{(k)}, \mu_{1}^{(k)}, \hat{\mu}_{1}^{(k)}\right)\right) \subset E_{1}^{+}$ such that $\mathcal{A} x^{(k)} \rightarrow s=\left(s_{1}, s_{2}\right)$ and $\left\langle x^{(k)}, c\right\rangle_{1} \rightarrow t$. Let us prove that $(s, t)=\left(\mathcal{A} x^{*},\left\langle x^{*}, c\right\rangle_{1}\right)$ for some $x^{*}=\left(\mu_{0}^{*}, \mu_{1}^{*}, \hat{\mu}_{1}^{*}\right) \in E_{1}^{+}$. As $c=(0,1,0)$, one has $\left\|\mu_{1}^{(k)}\right\|_{\mathrm{TV}}=\int_{\mathbf{B}} \mu_{1}^{(k)}(d \mathbf{y}) \rightarrow t(\geq$ 0 ), thus $\sup _{k}\left\|\mu_{1}^{(k)}\right\|_{\mathrm{TV}}<\infty$. Therefore there is a subsequence (denoted by the same indices) $\left(\mu_{1}^{(k)}\right)$ which converges to $\mu_{1}^{*} \in \mathcal{M}_{+}(\mathbf{B})$ for the weak-star topology. In particular $\left\|\mu_{1}^{*}\right\|_{T V}=t$. Hence from $\hat{\mu}_{1}^{(k)}+\mu_{1}^{(k)} \rightarrow s_{2}$ one deduces that $\hat{\mu}_{1}^{(k)} \rightarrow s_{2}-\mu_{1}^{*}$ for the weakstar topology. But then we also have $-f_{\#} \mu_{0}^{(k)} \rightarrow s_{1}-\mu_{1}^{*}$ in the weak-star topology of $\mathcal{M}(\mathbf{B})$. Therefore $-s_{1}+\mu_{1}^{*}$ is a positive measure. So let $\mu_{0}^{*}$ be such that $f_{\#} \mu_{0}^{*}=-s_{1}+\mu_{1}^{*}$ guaranteed to exist since we have seen that $f_{\#}\left(\mathcal{M}_{+}(S)\right)$ is weak-star closed. Then we have $\mathcal{A}\left(x^{*}\right)=\left(s_{1}, s_{2}\right)$ and $\left\langle x^{*}, c\right\rangle=t$, the desired result.

\subsection{Practical computation using semidefinite programming}

For each $r \geq r_{\min }^{(2)}:=\max \left\{\left\lceil r_{1}^{\mathbf{S}} / d\right\rceil, \ldots,\left\lceil r_{n}^{\mathbf{S}} / d\right\rceil, r_{1}^{\mathbf{B}}, \ldots, r_{n^{\mathbf{B}}}^{\mathbf{B}}\right\}$, let $\mathbf{z}_{0}=\left(z_{0 \beta}\right)_{\beta \in \mathbb{N}_{2 r}^{m}}$ be the finite sequence of moments up to degree $2 r$ of measure $\mu_{0}$. Similarly, let $\mathbf{z}_{1}$ and $\hat{\mathbf{z}}_{1}$ stand for the sequences of moments up to degree $2 r$, respectively associated with $\mu_{1}$ and $\hat{\mu}_{1}$. Problem 17] can be relaxed with the following semidefinite program:

$$
\begin{aligned}
p_{r}^{*}:=\sup _{\mathbf{z}_{0}, \mathbf{z}_{1}, \hat{\mathbf{z}}_{1}} & z_{10} \\
\text { s.t. } & z_{1 \beta}+\hat{z}_{1 \beta}=z_{\beta}^{\mathbf{B}}, L_{\mathbf{z}_{0}}\left(f(\mathbf{x})^{\beta}\right)=z_{1 \beta}, \quad \forall \beta \in N_{2 r}^{m}, \\
& \mathbf{M}_{r d-r_{j}^{\mathbf{S}}}\left(g_{j}^{\mathbf{S}} \mathbf{z}_{0}\right) \succeq 0, \quad j=0, \ldots, n^{\mathbf{S}}, \\
& \mathbf{M}_{r-r_{j}^{\mathbf{B}}}\left(g_{j}^{\mathbf{B}} \mathbf{z}_{1}\right) \succeq 0, \mathbf{M}_{r-r_{j}^{\mathbf{B}}}\left(g_{j}^{\mathbf{B}} \hat{\mathbf{z}}_{1}\right) \succeq 0, \quad j=0, \ldots, n^{\mathbf{B}} .
\end{aligned}
$$

Consider also the following semidefinite program, which is a strengthening of problem (22) and also the dual of problem 25):

$$
\begin{aligned}
d_{r}^{*}:=\inf _{v, w} & \sum_{\beta \in \mathbb{N}_{2 r}^{m}} w_{\beta} z_{\beta}^{\mathbf{B}} \\
\text { s.t. } & v \circ f \in \mathbf{Q}_{r d}(\mathbf{S}), \\
& w-1-v \in \mathbf{Q}_{r}(\mathbf{B}), \\
& w \in \mathbf{Q}_{r}(\mathbf{B}), \\
& v, w \in \mathbb{R}_{2 r}[\mathbf{y}] .
\end{aligned}
$$

Theorem 4.4. Let $r \geq r_{\min }^{(2)}$ and suppose that both $\mathbf{F}$ and $\mathbf{B} \backslash \mathbf{F}$ have nonempty interior and that Assumption 2.1 holds. Then: 
1. $p_{r}^{*}=d_{r}^{*}$, i.e. there is no duality gap between the semidefinite program (24) and its dual (25).

2. The semidefinite program (25) has an optimal solution $\left(v_{r}, w_{r}\right) \in \mathbb{R}_{2 r}[\mathbf{y}] \times \mathbb{R}_{2 r}[\mathbf{y}]$, and the sequence $\left(w_{r}\right)$ converges to $\mathbf{1}_{\mathbf{F}}$ in $L_{1}$ norm on $\mathbf{B}$ :

$$
\lim _{r \rightarrow \infty} \int\left|w_{r}(\mathbf{y})-\mathbf{1}_{\mathbf{F}}(\mathbf{y})\right| \lambda_{\mathbf{B}}(d \mathbf{y})=0 .
$$

3. Defining the set

$$
\mathbf{F}_{r}^{2}:=\left\{\mathbf{y} \in \mathbf{B}: w_{r}(\mathbf{y}) \geq 1\right\}
$$

its holds that

$$
\mathbf{F}_{r}^{2} \supset \mathbf{F}
$$

and

$$
\lim _{r \rightarrow \infty} \operatorname{vol}\left(\mathbf{F}_{r}^{2} \backslash \mathbf{F}\right)=0
$$

Proof.

1. Let $\mu_{1}=\lambda_{\mathbf{F}}$, let $\mu_{0}$ be such that $f_{\#} \mu_{0}=\mu_{1}$ as in Lemma 4.1, and let $\hat{\mu}_{1}=\lambda_{\mathbf{B}}-\mu_{1}$ so that $\left(\mu_{0}, \mu_{1}, \hat{\mu}_{1}\right)$ is feasible for LP 17$)$. Given $r \geq r_{\min }^{(2)}$, let $\mathbf{z}_{0}, \mathbf{z}_{1}$ and $\hat{\mathbf{z}}_{1}$ be the sequences of moments up to degree $2 r$ of $\mu_{0}, \mu_{1}$ and $\hat{\mu}_{1}$, respectively. Clearly, $\left(\mathbf{z}_{0}, \mathbf{z}_{1}, \hat{\mathbf{z}}_{1}\right)$ is feasible for program (24). Then, as in the proof of the first item of Theorem 3.3, the optimal solution set of the program (24) is nonempty and bounded, which by [Trn05] implies that there is no duality gap between the semidefinite program (25) and its dual (24).

2. Now, one shows that $\left(\mathbf{z}_{0}, \mathbf{z}_{1}, \hat{\mathbf{z}}_{1}\right)$ is strictly feasible for program (24). Using the fact that

(a) $\mathbf{F}$ (resp. $\mathbf{B} \backslash \mathbf{F}$ ) has nonempty interior,

(b) $\mathbf{z}_{1}$ (resp. $\hat{\mathbf{z}_{1}}$ ) is the moment sequence of $\mu_{1}$ (resp. $\hat{\mu}_{1}$ ),

one has $\mathbf{M}_{r}\left(g_{j}^{\mathbf{B}} \mathbf{z}_{1}\right) \succ 0\left(\operatorname{resp} . \mathbf{M}_{r}\left(g_{j}^{\mathbf{B}} \hat{\mathbf{z}}_{1}\right) \succ 0\right)$, for each $j=0, \ldots, n^{\mathbf{B}}$. Moreover, $\mathbf{M}_{r}\left(g_{j}^{\mathbf{S}} \mathbf{z}_{0}\right) \succ 0$, for all $j=0, \ldots, n^{\mathbf{S}}$. Otherwise, assume that there exists a nontrivial vector $\mathbf{q}$ such that $\mathbf{M}_{r}\left(g_{j}^{\mathbf{S}} \mathbf{z}_{0}\right) \mathbf{q}=0$ for some $j$. As $\mathbf{F}$ has nonempty interior, it contains an open set $\mathbf{A} \subset \mathbb{R}^{m}$. By continuity of $f$, the set $f^{-1}(\mathbf{A}):=\{\mathbf{x} \in \mathbf{S}$ : $f(\mathbf{x}) \in \mathbf{A}\}$ is an open set of $\mathbf{S}$ and $\mu_{0}\left(f^{-1}(\mathbf{A})\right)=\mu_{1}(\mathbf{A})>0$. Then,

$$
0=\left\langle\mathbf{q}, \mathbf{M}_{r}\left(g_{j}^{\mathbf{S}} \mathbf{z}_{0}\right) \mathbf{q}\right\rangle=\int_{\mathbf{S}} q(\mathbf{x})^{2} g_{j}^{\mathbf{S}}(\mathbf{x}) d \mu_{0}(\mathbf{x}) \geq \int_{f^{-1}(\mathbf{A})} q(\mathbf{x})^{2} g_{j}^{\mathbf{S}}(\mathbf{x}) d \mu_{0}(\mathbf{x}),
$$

which yields $q(\mathbf{x})^{2} g_{j}^{\mathbf{S}}(\mathbf{x})=0$ on the open set $f^{-1}(\mathbf{A})$, leading to a contradiction.

Therefore, as for the proof of the second item of Theorem 3.3, we conclude that the semidefinite program 25 has an optimal solution $\left(v_{r}, w_{r}\right) \in \mathbb{R}_{2 r}[\mathbf{y}] \times \mathbb{R}_{2 r}[\mathbf{y}]$. 
Next, one proves that there exists a sequence of polynomials $\left(w_{k}\right)_{k \in \mathbb{N}} \subset \mathbb{R}[\mathbf{y}]$ such that $w_{k}(\mathbf{y}) \geq \mathbf{1}_{\mathbf{F}}(\mathbf{y})$, for all $\mathbf{y} \in \mathbf{B}$ and such that

$$
\lim _{k \rightarrow \infty} \int\left|w_{k}(\mathbf{y})-\mathbf{1}_{\mathbf{F}}(\mathbf{y})\right| \lambda_{\mathbf{B}}(d \mathbf{y})=0 .
$$

The set $\mathbf{F}$ being closed, the indicator function $\mathbf{1}_{\mathbf{F}}$ is upper semi-continuous and bounded, so there exists a non-increasing sequence of bounded continuous functions $h_{k}: \mathbf{B} \rightarrow \mathbb{R}$ such that $h_{k}(\mathbf{y}) \downarrow \mathbf{1}_{\mathbf{F}}(\mathbf{y})$, for all $\mathbf{y} \in \mathbf{B}$, as $k \rightarrow \infty$. Using the Monotone Convergence Theorem, $h_{k} \rightarrow \mathbf{1}_{\mathbf{F}}$ for the $L_{1}$ norm. By the StoneWeierstrass Theorem, there exists a sequence of polynomials $\left(w_{k}^{\prime}\right)_{k \in \mathbb{N}} \subset \mathbb{R}[\mathbf{y}]$, such that $\sup _{\mathbf{y} \in \mathbf{B}}\left|w_{k}^{\prime}(\mathbf{y})-h_{k}(\mathbf{y})\right| \leq 1 / k$. The polynomial $w_{k}:=w_{k}^{\prime}+1 / k$ satisfies $w_{k}>h_{k} \geq \mathbf{1}_{\mathbf{F}}$ and (27) holds.

Let us define $\tilde{w}_{k}:=w_{k}+\epsilon /(2 \operatorname{vol} \mathbf{B}), \tilde{v}_{k}:=w_{k}-1$. Next, for $r \in \mathbb{N}$ large enough, one proves that $\left(\tilde{v}_{k}, \tilde{w}_{k}\right)$ is a feasible solution of (25). Using the fact that $\tilde{w}_{k}>w_{k}>\mathbf{1}_{\mathbf{F}}$, one has $\tilde{w}_{k} \in \mathbf{Q}_{r}(\mathbf{B})$, as a consequence of Putinar's Positivstellensatz. For each $\mathbf{x} \in \mathbf{S}, \tilde{v}_{k}(f(\mathbf{x}))=w_{k}(\mathbf{y})-1>\mathbf{1}_{\mathbf{F}}(f(\mathbf{x}))-1+\epsilon /(2 \operatorname{vol} \mathbf{B})>0$, so $\tilde{v}_{k} \circ f$ lies in $\mathbf{Q}_{r d}(\mathbf{S})$. Similarly, $\tilde{w}_{k}-\tilde{v}_{k}-1 \in \mathbf{Q}_{r}(\mathbf{B})$. Then, one concludes using the same arguments as for (14) in the proof of the second item of Theorem 3.3

3. Let $\mathbf{y} \in \mathbf{F}$. There exists $\mathbf{x} \in \mathbf{S}$ such that $\mathbf{y}=f(\mathbf{x})$. Let $\left(v_{r}, w_{r}\right) \in \mathbb{R}_{2 r}[\mathbf{y}] \times \mathbb{R}_{2 r}[\mathbf{y}]$ be an optimal solution of (25). By feasibility, $w_{r}(\mathbf{y})-1 \geq v_{r}(\mathbf{y})=v_{r}(f(\mathbf{x})) \geq 0$. Thus, $\mathbf{F}_{r}^{2} \supset \mathbf{F}$. Finally, the proof of the convergence in volume is analogous to the proof of (6) in Theorem 3.2 .

\section{Computational considerations}

\subsection{Complexity analysis and lifting strategy}

\subsubsection{Method 1}

First, consider the semidefinite program (13) of Method 1. For $r \geq r_{\min }^{(1)}$, the number of variables $n^{(1)}$ (resp. size of semidefinite matrices $m^{(1)}$ ) of problem (13) satisfies:

$$
n^{(1)} \leq\left(\begin{array}{c}
n+m+2 r \\
2 r
\end{array}\right) \text {. }
$$

Problem (13) involves $\left(n^{\mathbf{B}}+n^{\mathbf{S}}+1\right)$ semidefinite constraints of size $m^{(1)}$ bounded as follows:

$$
m^{(1)} \leq\left(\begin{array}{c}
n+m+r \\
r
\end{array}\right) .
$$




\subsubsection{Method 2}

Now, consider the semidefinite program (24). For $r \geq r_{\min }^{(2)}$, the number of variables $n^{(2)}$ of Problem (24) satisfies:

$$
n^{(2)} \leq\left(\begin{array}{c}
n+2 r d \\
2 r d
\end{array}\right)+2\left(\begin{array}{c}
m+2 r \\
2 r
\end{array}\right) .
$$

Problem (24) also involves $\left(n^{\mathbf{S}}+1\right)$ semidefinite constraints of size at most $\left(\begin{array}{c}n+r d \\ r d\end{array}\right)$ and $2\left(n^{\mathbf{B}}+1\right)$ semidefinite constraints of size at most $\left(\begin{array}{c}m+r \\ r\end{array}\right)$.

Due to the dependence on the degree $d$ of the polynomial application, one observes that the number of variables (resp. constraints) can quickly become large if $d$ is not small. An alternative formulation to limit the blowup of these relaxations is obtained by considering $y_{1}, \ldots, y_{m}$ as "lifting" variables, respectively associated with $f_{1}, \ldots, f_{m}$, together with the following $2 m$ additional constraints:

$$
g_{n_{\mathbf{S}}+j}^{\mathbf{S}}(\mathbf{x}, \mathbf{y}):=y_{j}-f_{j}(\mathbf{x}), \quad g_{n_{\mathbf{S}+2 j}}^{\mathbf{S}}(\mathbf{x}, \mathbf{y}):=f_{j}(\mathbf{x})-y_{j}, \quad j=1, \ldots, m .
$$

By considering the basic compact semi-algebraic set $\mathbf{S}_{\text {lift }} \subset \mathbb{R}^{n+m}$ given by

$$
\mathbf{S}_{\text {lift }}:=\left\{(\mathbf{x}, \mathbf{y}) \in \mathbf{K}: g_{n}^{\mathbf{S}_{+1}}(\mathbf{x}, \mathbf{y}) \geq 0, \ldots, g_{n}^{\mathbf{s}_{+2 m}}(\mathbf{x}, \mathbf{y}) \geq 0\right\},
$$

problem 25) becomes:

$$
\begin{array}{ll}
\inf _{v, w} & \sum_{\beta \in \mathbb{N}_{2 r}^{m}} w_{\beta} z_{\beta}^{\mathbf{B}} \\
\text { s.t. } & v \in \mathbf{Q}_{r}\left(\mathbf{S}_{\text {lift }}\right), \\
& w-1-v \in \mathbf{Q}_{r}(\mathbf{B}), \\
& w \in \mathbf{Q}_{r}(\mathbf{B}), \\
& v, w \in \mathbb{R}_{2 r}[\mathbf{y}],
\end{array}
$$

which is actually equivalent to the following problem:

$$
\begin{array}{ll}
\inf _{w} & \sum_{\beta \in \mathbb{N}_{2 r}^{m}} w_{\beta} z_{\beta}^{\mathbf{B}} \\
\text { s.t. } & w-1 \in \mathbf{Q}_{r}\left(\mathbf{S}_{\text {lift }}\right), \\
& w \in \mathbf{Q}_{r}(\mathbf{B}), \\
& w \in \mathbb{R}_{2 r}[\mathbf{y}] .
\end{array}
$$

The minimal relaxation order of problem $(30)$ is $r_{\text {lift }}^{(2)}:=\max \left\{\left\lceil\frac{d}{2}\right\rceil, r_{1}^{\mathbf{S}}, \ldots, r_{n^{\mathrm{S}}}^{\mathbf{S}}, r_{1}^{\mathbf{B}}, \ldots, r_{n^{\mathrm{B}}}^{\mathbf{B}}\right\}$ and the number of variables $n_{\text {lift }}^{(2)}$ is bounded as follows:

$$
n_{\text {lift }}^{(2)} \leq\left(\begin{array}{c}
n+m+2 r \\
2 r
\end{array}\right)+\left(\begin{array}{c}
m+2 r \\
2 r
\end{array}\right) .
$$

Problem (30) involves $\left(n^{\mathbf{S}}+2 m+1\right)$ semidefinite constraints of size at most $\left(\begin{array}{c}n+m+r \\ r\end{array}\right)$ and $\left(n^{\mathbf{B}}+1\right)$ semidefinite constraints of size at most $\left(\begin{array}{c}m+r \\ r\end{array}\right)$. When $m$ is small and $d$ is large, this seems to be a suitable choice to reduce the computational cost of the semidefinite program (24). Experimental results described further (see Table 1 in Section 6.1 and Table 2 in Section 6.4) agree with this observation. 


\section{$5.2 \quad$ Exploiting sparsity}

As explained above, both Method 1 and Method 2 are computationally demanding in general. However, if the polynomials $\mathbf{x} \mapsto f_{j}(\mathbf{x}),(j=1, \ldots, m)$ have some structured sparsity, then one can still exploit sparsity in a way similar to the one described in WKKM06, Las06 to handle problems in higher dimensions. In particular, let $\{1, \ldots, n\}$ be the union $\bigcup_{j=1}^{m} I_{j}$ of subsets $I_{j} \subseteq\{1, \ldots, n\}$ and assume that for each $j=1, \ldots, m$, the polynomial $f_{j}$ involves only variables $\left\{x_{i} \mid i \in I_{j}\right\}$. One also suppose that the collection $\left\{I_{1}, \ldots, I_{m}\right\}$ satisfies the so-called running intersection property:

Definition 5.1. The collection $\left\{I_{1}, \ldots, I_{m}\right\}$ of subsets $I_{j} \subseteq\{1, \ldots, n\}$ satisfies the running intersection property if the following holds for each $j=1, \ldots, m-1$ :

$$
I_{j+1} \cap \bigcup_{k=1}^{j} I_{k} \subseteq I_{l} \quad \text { for some } l \leq j .
$$

The following assumption allows to apply the sparse representation result of [Las06, Corollary 3.9] while using either Method 1 or Method 2.

Assumption 5.2. The index set $\{1, \ldots, n\}$ is partitioned into $m$ disjoint sets $I_{j}, j=$ $1, \ldots, m$ so that:

1. The collection $\left\{I_{1}, \ldots, I_{m}\right\}$ satisfies the running intersection property.

2. For each $j=1, \ldots, n^{\mathbf{S}}$, there exists some $k_{j}$ such that the polynomial $g_{j}^{\mathbf{S}}$ in (1) involves only variables $\left\{x_{i} \mid i \in I_{k_{j}}\right\}$.

3. In the definition (1) of $\mathbf{S}$, we replace the inequality constraint $N^{\mathbf{S}}-\|\mathbf{x}\|_{2}^{2} \geq 0$ by the $m$ quadratic constraints:

$$
N_{j}-\sum_{i \in I_{j}} x_{i}^{2} \geq 0, \quad j=1, \ldots, m .
$$

For each $j=1, \ldots, m$, index the variable $y_{j}$ by $n+j$ and define $I_{j}^{(1)}:=I_{j} \bigcup\{n+1, \ldots, n+$ $m\}$.

Proposition 5.3. Under Assumption 5.2, the collection $\left\{I_{1}^{(1)}, \ldots, I_{m}^{(1)}\right\}$ of subsets $I_{j}^{(1)} \subseteq$ $\{1, \ldots, n, n+1, \ldots, n+m\}$ satisfies the running intersection property.

Proof. The collection $\left\{I_{1}, \ldots, I_{m}\right\}$ of subsets $I_{j} \subseteq\{1, \ldots, n\}$ satisfies the running intersection property. For each $j=1, \ldots, m-1$, there exists $l \leq j$ such that $I_{j+1} \cap \bigcup_{k=1}^{j} I_{k} \subseteq I_{l}$. Thus, $I_{j+1}^{(1)} \cap \bigcup_{k=1}^{j} I_{k}^{(1)}=\left(I_{j+1} \cap \bigcup_{k=1}^{j} I_{k}\right) \bigcup\{n+1, \ldots, n+m\} \subseteq I_{l} \bigcup\{n+1, \ldots, n+m\}=I_{l}^{(1)}$, the desired result.

Then Assumption 5.2 allows one to apply the sparse representation result of Las06. Corollary 3.9] to the semidefinite program (12) associated with Method 1. Indeed, observe that the polynomial $(\mathbf{x}, \mathbf{y}) \mapsto h_{f}(\mathbf{x}, \mathbf{y})$ can be decomposed as $h_{f}=\sum_{j=1}^{m} h_{f_{j}}$, where for 
each $j=1, \ldots, m$, the polynomial $h_{f_{j}}$ involves only the variables $\left\{x_{i} \mid i \in I_{j}\right\}$ (the same variables involved in $f_{j}$ ) and $\mathbf{y}$.

Under Assumption 5.2, this sparse representation result can also be applied for the semidefinite program (30) associated with the lifting variant of Method 2 described in Section 5.1.2. This is due to the fact that for each $j=1, \ldots, m$, the polynomials $g_{n}^{\mathbf{S}} \mathbf{s}_{+j}$ and $g_{n}^{\mathbf{s}}{ }_{+2 j}$ involve only the variables $\left\{x_{i} \mid i \in I_{j}\right\}$ and $\mathbf{y}$.

\section{Application examples}

Here we present some application examples together with numerical results. In particular, this section illustrates that our methodology is a unified framework which can tackle important special cases: semi-algebraic set projections (Section 6.2) and Pareto curves approximations (Section 6.3). Moreover, the framework can be extended to approximate images of semi-algebraic sets under semi-algebraic applications (Section 6.4).

The numerical results are given after solving either the semidefinite program (12) for Method 1, the semidefinite program 25) for Method 2 or the semidefinite program (30) for the lifting variant of Method 2, with the YALMIP toolbox [Lö04 for MatlaB. As explained in Section 5.1.2, the outer approximations obtained by Method 2 and its lifting variant are the same, but their semidefinite formulations differ.

Benchmarks are performed on an Intel Core i5 CPU $(2.40 \mathrm{GHz})$ with YALMIP interfaced with the semidefinite programming solver MosEK [AA00].

\subsection{Polynomial image of semi-algebraic sets}

Example 1. Consider the image of the two-dimensional unit ball $\mathbf{S}:=\left\{\mathbf{x} \in \mathbb{R}^{2}:\|\mathbf{x}\|_{2}^{2} \leq\right.$ 1 \} under the polynomial application $f(\mathbf{x}):=\left(x_{1}+x_{1} x_{2}, x_{2}-x_{1}^{3}\right) / 2$. We choose $\mathbf{B}=\mathbf{S}$ since it can be checked that $\mathbf{F}=f(\mathbf{S}) \subset \mathbf{B}$.

On Figure 1 resp. 2, we represent in light gray the outer approximations $\mathbf{F}_{r}^{1}$ resp. $\mathbf{F}_{r}^{2}$ of $\mathbf{F}$ obtained by Method 1 resp. 2, for increasing values of the relaxation order $r$. On each figure, the black dots correspond to the image set of the points obtained by uniform sampling of $\mathbf{S}$ under $f$. We observe that the approximations behave well around the locally convex parts of the boundary of $\mathbf{F}$, and that it is not straightforward to decide whether Method 1 or Method 2 provides the best approximations.

We indicate in Table 1 the data related to the semidefinite programs solved by MosEK to compute approximations of increasing degrees, while using Method 1, Method 2 and Method 2 with the lifting strategy (see Section 5.1.2 for more details). For each problem, "vars" stands for the total number of variables and "size" stands for the size of the semidefinite matrices. The computational timings of Method 2 with the lifting strategy are similar to those of Method 1, for $r=1, \ldots, 5$. However, for $r=6$ the size of the problem is significantly smaller with Method 1. 


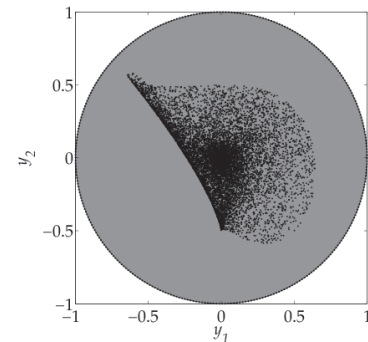

(a) $r=1$

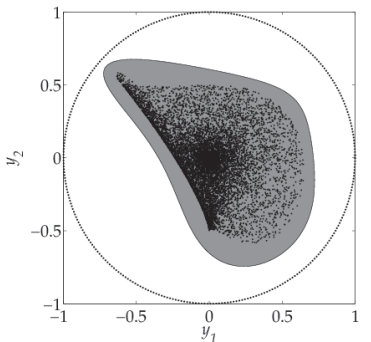

(b) $r=2$

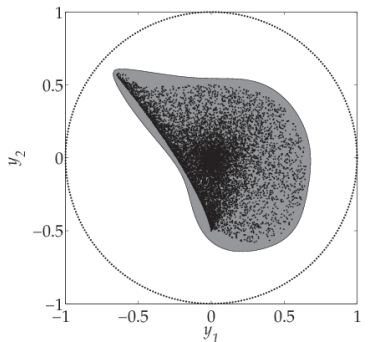

(c) $r=3$

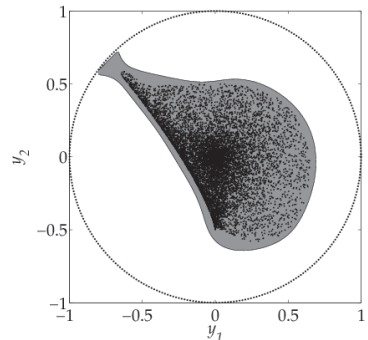

(d) $r=4$

Figure 1: Outer approximations $\mathbf{F}_{r}^{1}$ (light gray) of $\mathbf{F}$ (black dot samples) for Example 1 . for $r=1,2,3,4$.

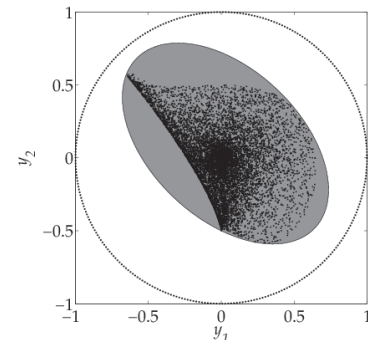

(a) $r=1$

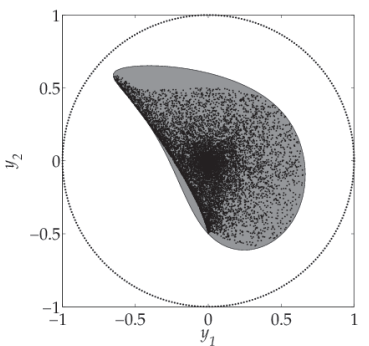

(b) $r=2$

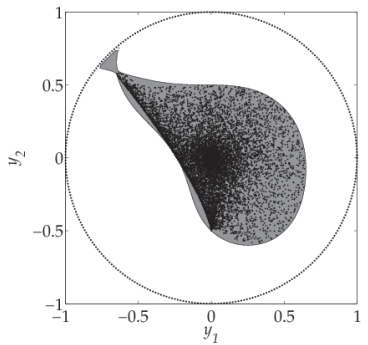

(c) $r=3$

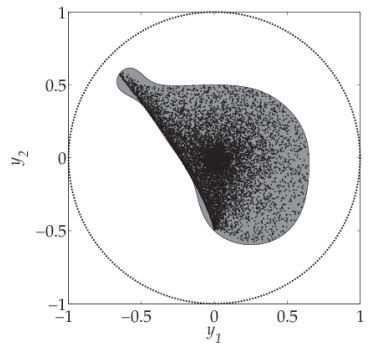

(d) $r=4$

Figure 2: Outer approximations $\mathbf{F}_{r}^{2}$ (light gray) of $\mathbf{F}$ (black dot samples) for Example 1 . for $r=1,2,3,4$.

Table 1: Comparison of timing results for Example 1

\begin{tabular}{l|c|cccccc}
\hline \multicolumn{2}{c|}{ relaxation order $r$} & 1 & 2 & 3 & 4 & 5 & 6 \\
\hline \multirow{3}{*}{ Method 1 } & vars & 40 & 212 & 1039 & 4211 & 14028 & 40251 \\
& size & 30 & 111 & 350 & 915 & 1991 & 3822 \\
& time (s) & 0.64 & 0.72 & 0.77 & 1.69 & 8.22 & 40.37 \\
\hline \multirow{3}{*}{ Method 2 } & vars & 286 & 2140 & 8241 & 22720 & 51166 & 100626 \\
& size & 129 & 471 & 1029 & 1803 & 2793 & 3999 \\
& time (s) & 0.65 & 0.74 & 1.54 & 3.4 & 12.89 & 43.74 \\
\hline \multirow{2}{*}{ Method 2 } & vars & 51 & 308 & 1499 & 5882 & 19546 & 56710 \\
with lifting & size & 32 & 157 & 536 & 1411 & 3128 & 6127 \\
& time (s) & 0.58 & 0.66 & 0.68 & 1.93 & 10.07 & 63.88 \\
\hline
\end{tabular}

\subsection{Projections of semi-algebraic sets}

For $n \geq m$, we focus on the special case of projections. Let $f$ be the projection of $\mathbf{S}$ with respect to the $m$ first coordinates, i.e. $f(\mathbf{x}):=\left(x_{1}, \ldots, x_{m}\right)$. It turns out that in this case, the semidefinite program (30) associated to the lifting variant of Method 2, has the 
following simpler formulation:

$$
\begin{array}{ll}
\inf _{w} & \sum_{\beta \in \mathbb{N}_{2 r}^{m}} w_{\beta} z_{\beta}^{\mathbf{B}} \\
\text { s.t. } & w-1 \in \mathbf{Q}_{r}(\mathbf{S}), \\
& w \in \mathbf{Q}_{r}(\mathbf{B}), \\
& w \in \mathbb{R}_{2 r}\left[x_{1}, \ldots, x_{m}\right] .
\end{array}
$$

Example 2. Consider the projection $\mathbf{F}$ on the first two coordinates of the semi-algebraic set $\mathbf{S}:=\left\{\mathbf{x} \in \mathbb{R}^{3}:\|x\|_{2}^{2} \leq 1,1 / 4-\left(x_{1}+1 / 2\right)^{2}-x_{2}^{2} \leq 0,1 / 9-\left(x_{1}-1 / 2\right)^{4}-x_{2}^{4} \leq 0\right\}$, which belongs to $\mathbf{B}:=\left\{x \in \mathbb{R}^{2}:\|x\|_{2}^{2} \leq 1\right\}$.

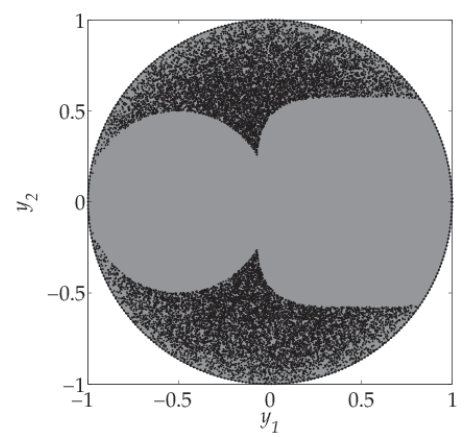

(a) $r=2$

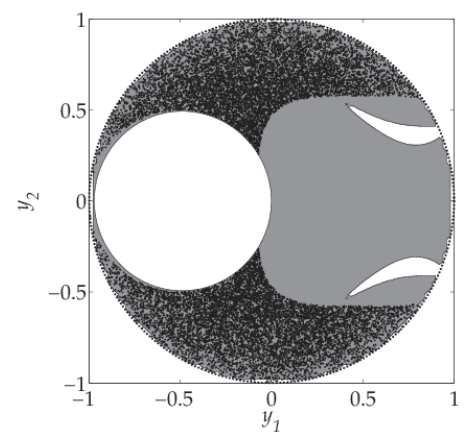

(b) $r=3$

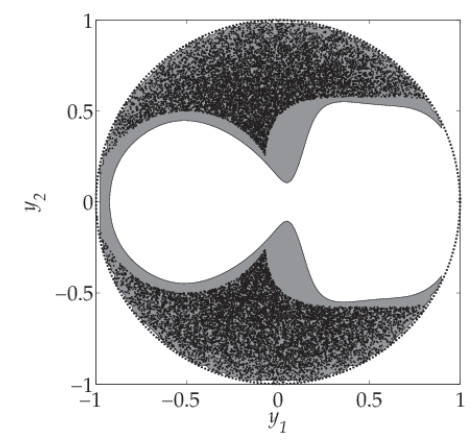

(c) $r=4$

Figure 3: Outer approximations $\mathbf{F}_{r}^{1}$ (light gray) of $\mathbf{F}$ (black dot samples) for Example 2 , for $r=2,3,4$.

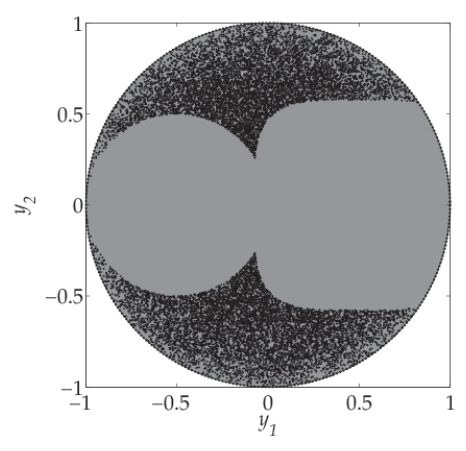

(a) $r=2$

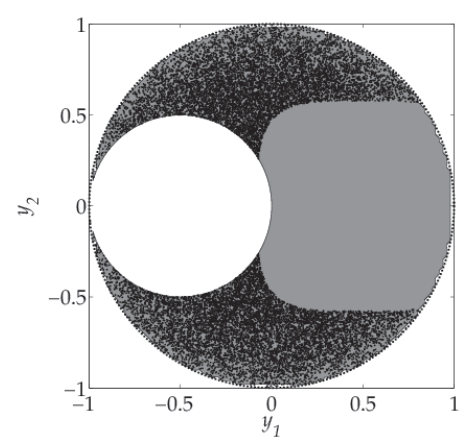

(b) $r=3$

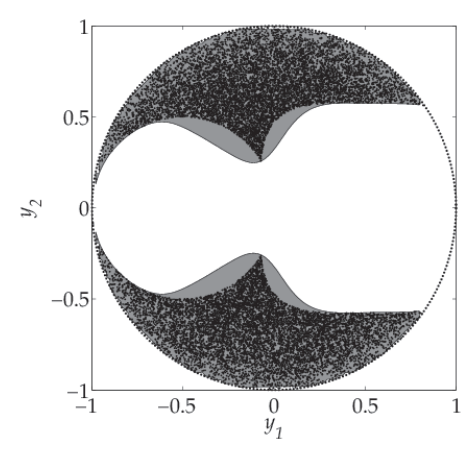

(c) $r=4$

Figure 4: Outer approximations $\mathbf{F}_{r}^{2}$ (light gray) of $\mathbf{F}$ (black dot samples) for Example 2 , for $r=2,3,4$.

Figure 3 resp. 4 displays approximation of the projection of $\mathbf{S}$ on the first two coordinates with Method 1 resp. 2. 


\subsection{Approximating Pareto curves}

In [MHL14, we propose a method to approximate Pareto curves associated with bicriteria polynomial optimization problems $\min _{\mathbf{x} \in \mathbf{S}}\left\{\left(f_{1}(\mathbf{x}), f_{2}(\mathbf{x})\right)\right\}$. The image space $\mathbb{R}^{2}$ is partially ordered with the positive orthant $\mathbb{R}_{+}^{2}$, that is, for every $\mathbf{y}_{1}, \mathbf{y}_{2} \in \mathbb{R}^{2}, \mathbf{y}_{1} \geq \mathbf{y}_{2}$ stands for $\mathbf{y}_{2}-\mathbf{y}_{1} \in \mathbb{R}_{+}^{2}$. A point $\overline{\mathbf{x}}$ is called a weakly Edgeworth-Pareto optimal point, when there is no $\mathbf{x} \in \mathbf{S}$ such that $f_{j}(\mathbf{x})<f_{j}(\overline{\mathbf{x}}), j=1,2$. The Pareto curve is the set of weakly Edgeworth-Pareto optimal points. For more details on multicriteria optimization, we refer the interested reader to [Jah10] and the references therein.

The methodology of [MHL14 consists of reformulating the initial bicriteria optimization problem to use a hierarchy of semidefinite approximations for parametric polynomial optimization problems. Then, one can apply the framework developed in [Las10] and build a hierarchy of semidefinite programs, allowing to approximate as closely as desired the Pareto curve. Here we propose to study outer approximations of the set $\mathbf{F}=\left(f_{1}(\mathbf{S}), f_{2}(\mathbf{S})\right)$ since points along the boundary of a tight outer approximation are expected to be close to the Pareto curve.

Example 3. Let consider the two-dimensional nonlinear problem proposed in [WCSF01]: $\min _{\mathbf{x} \in \mathbf{S}}\left\{\left(f_{1}(\mathbf{x}), f_{2}(\mathbf{x})\right)\right\}$, with $f_{1}(\mathbf{x}):=\frac{\left(x_{1}+x_{2}-7.5\right)^{2}}{4}+\left(x_{2}-x_{1}+3\right)^{2}, f_{2}(\mathbf{x}):=x_{1}+x_{2}^{2}$ and $\mathbf{S}:=\left\{\mathbf{x} \in \mathbb{R}^{2}:-\left(x_{1}-2\right)^{3} / 2-x_{2}+2.5 \geq 0,-x_{1}-x_{2}+8\left(x_{2}-x_{1}+0.65\right)^{2}+3.85 \geq 0\right\}$. Instead of $f_{1}$, we consider $\tilde{f}_{1}:=\left(f_{1}(\mathbf{x})-a_{1}\right) /\left(b_{1}-a_{1}\right)$, where $a_{1}$ and $b_{1}$ are given by $a_{1}:=\min _{\mathbf{x} \in \mathbf{S}} f_{1}(\mathbf{x})$ and $b_{1}:=f_{1}(\overline{\mathbf{x}})$ with $\overline{\mathbf{x}}$ a solution of $\min _{\mathbf{x} \in \mathbf{S}} f_{2}(\mathbf{x})$. Similarly, we consider a scaled criterion $\tilde{f}_{2}$ defined from $f_{2}$. A preprocessing step consists in computing lower and upper bounds of the polynomial $f_{1}$ (resp. $f_{2}$ ) over $\mathbf{S}$ to define $\tilde{f}=\left(\tilde{f}_{1}, \tilde{f}_{2}\right)$. Doing so, one ensures that $\tilde{f}(\mathbf{S})$ is a subset of the unit ball $\mathbf{B}$ and the present methodology applies.

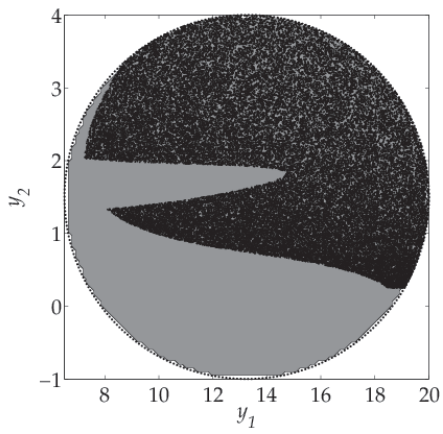

(a) $r=1$

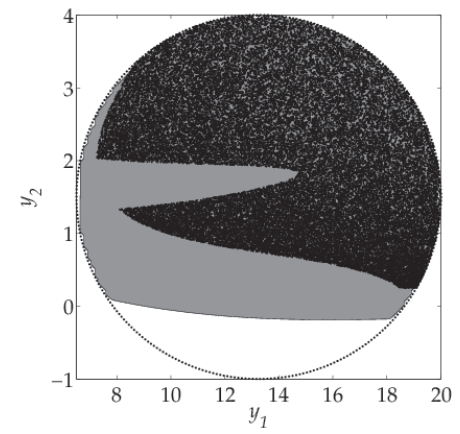

(b) $r=2$

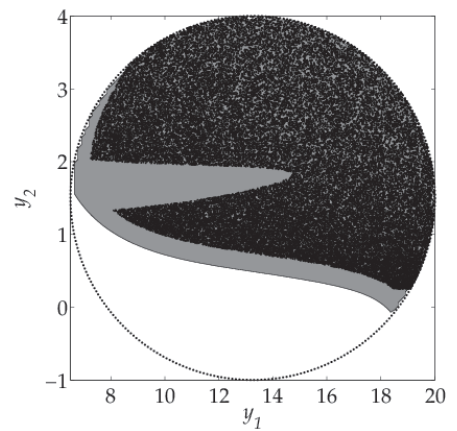

(c) $r=4$

Figure 5: Outer approximations $\mathbf{F}_{r}^{1}$ (light gray) of $\mathbf{F}$ (black dot samples) for Example 3 for $r=1,2,4$.

In this case, the Pareto curve is nonconvex and disconnected. As depicted in Figure 5 and Figure 6, it is difficult to obtain precise approximations of the whole image set, in particular for the subset $\mathbf{F} \cap \mathbf{B}_{0}$, with an ellipse $\mathbf{B}_{0}:=\left\{\mathbf{x} \in \mathbb{R}^{2}:\left(\left(x_{1}-13.7\right) / 1.7\right)^{2}+\right.$ $\left.\left(\left(x_{2}-1.8\right) / 0.5\right)^{2} \leq 1\right\}$. Figure 7 displays more precise outer approximations of degree 8 (a) and degree 10 (b). 


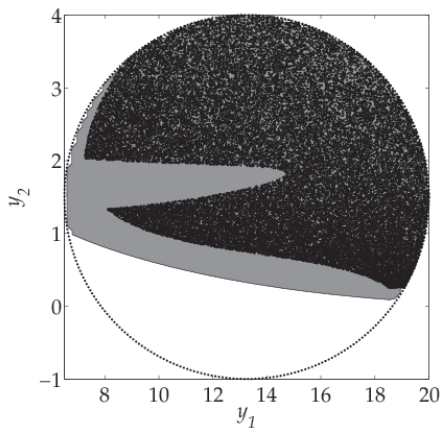

(a) $r=1$

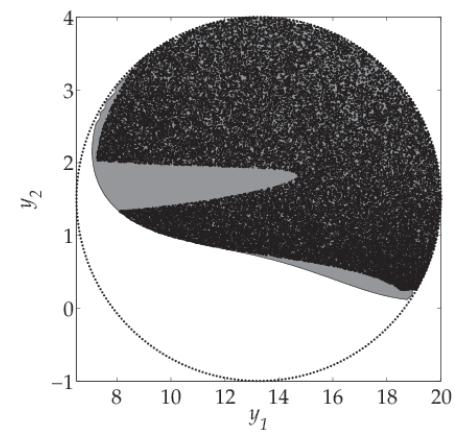

(b) $r=2$

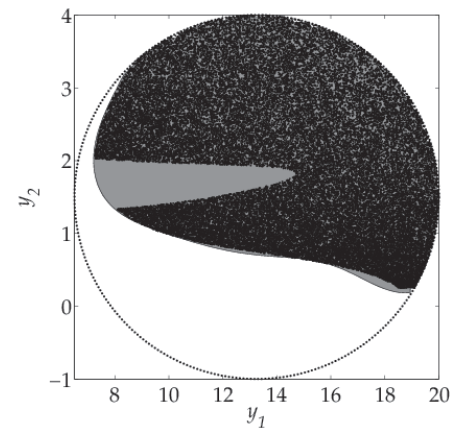

(c) $r=4$

Figure 6: Outer approximations $\mathbf{F}_{r}^{2}$ (light gray) of $\mathbf{F}$ (black dot samples) for Example 3 for $r=1,2,4$.

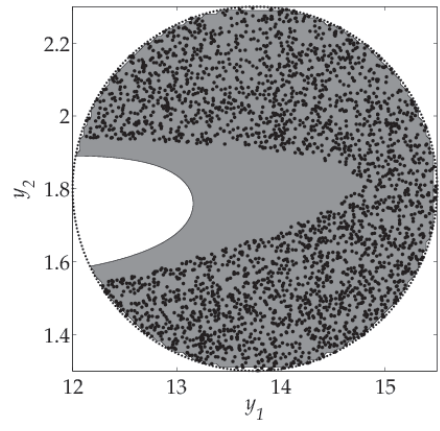

(a) $r=4$

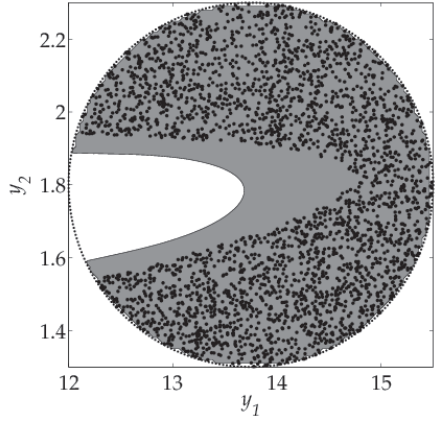

(b) $r=5$

Figure 7: Outer approximations $\mathbf{F}_{r}^{1}$ (light gray) of $\mathbf{F} \cap \mathbf{B}_{0}$ (black dot samples) for Example 3, for $r=4,5$.

\section{4 semi-algebraic image of semi-algebraic sets}

Given a semi-algebraic set $\mathbf{S}$ as in (1), Methods 1 and 2 can be extended to approximate the image of $\mathbf{S}$ under a semi-algebraic application $f=\left(f_{1}, \ldots, f_{m}\right)$. To do so, we follow [LP10] and introduce lifting variables to represent non-polynomial components involved in $f_{1}, \ldots, f_{m}$, as well as additional polynomial constraints.

Proceeding as in [LP10], for each semi-algebraic function $f_{j}$, one introduces additional variables $\mathbf{x}^{j}:=\left(x_{1}^{j}, \ldots, x_{t_{j}}^{j}\right)$ such that the graph $\left\{\left(\mathbf{x}, f_{j}(\mathbf{x})\right): \mathbf{x} \in \mathbf{S}\right\}=\left\{\left(\mathbf{x}, x_{t_{j}}^{j}\right)\right.$ : $\left.\left(\mathbf{x}, \mathbf{x}^{j}\right) \in \hat{\mathbf{S}}_{j}\right\}$ for some semi-algebraic set $\hat{\mathbf{S}}_{j} \subseteq \mathbb{R}^{n+t_{j}}$. In the end, one works with the lifted set $\hat{\mathbf{S}}:=\left\{\left(\mathbf{x}, \mathbf{x}^{1}, \ldots, \mathbf{x}^{m}\right):\left(\mathbf{x}, \mathbf{x}^{j}\right) \in \hat{\mathbf{S}}_{j}, j=1, \ldots, m\right\}$.

Example 4. Here, we consider the image of the two-dimensional unit ball $\mathbf{S}:=\left\{\mathbf{x} \in \mathbb{R}^{2}\right.$ : $\left.\|\mathbf{x}\|_{2}^{2} \leq 1\right\}$ under the semi-algebraic application $f(\mathbf{x}):=\left(\min \left(x_{1}+x_{1} x_{2}, x_{1}^{2}\right), x_{2}-x_{1}^{3}\right) / 3$. Remind that $2 \min (a, b)=a+b-|a-b|$, so that $2 \min \left(x_{1}+x_{1} x_{2}, x_{1}^{2}\right)=x_{1}+x_{1} x_{2}+$ $x_{1}^{2}-\left|x_{1}+x_{1} x_{2}-x_{1}^{2}\right|$. To handle the absolute value, we introduce an additional variable $x_{3}$ together with the equality constraint $x_{3}^{2}=\left(x_{1}+x_{1} x_{2}^{2}\right)^{2}$ and the inequality constraint $x_{3} \geq 0$. 


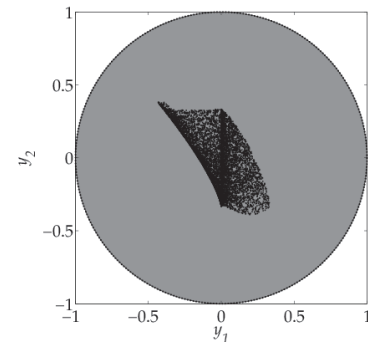

(a) $r=1$

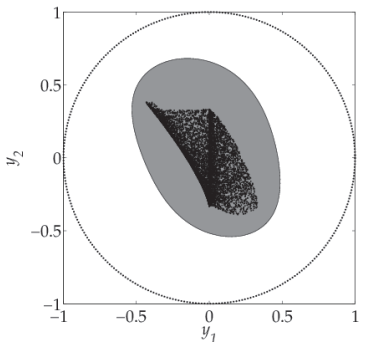

(b) $r=2$

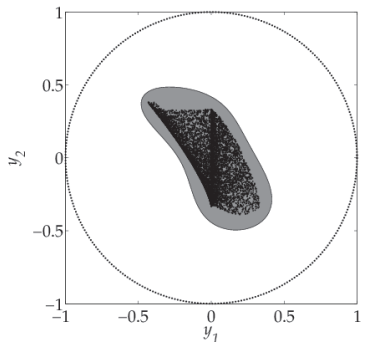

(c) $r=3$

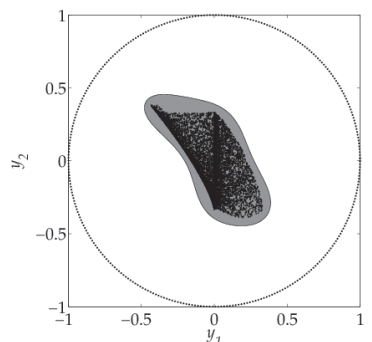

(d) $r=4$

Figure 8: Outer approximations $\mathbf{F}_{r}^{1}$ (light gray) of $\mathbf{F}$ (black dot samples) for Example 4 . for $r=1,2,3,4$.

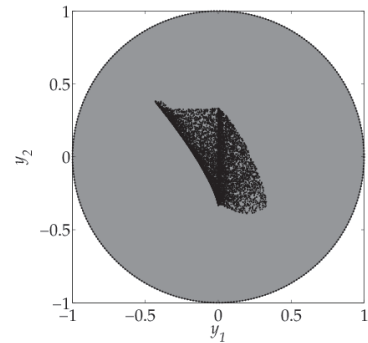

(a) $r=1$

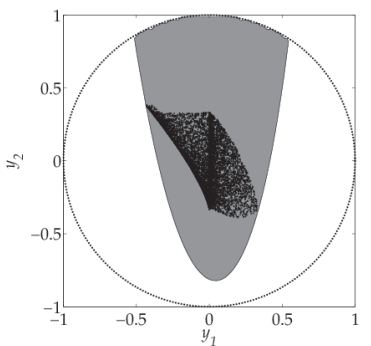

(b) $r=2$

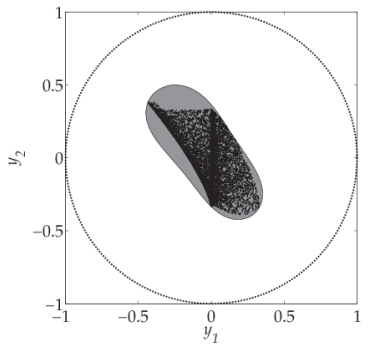

(c) $r=3$

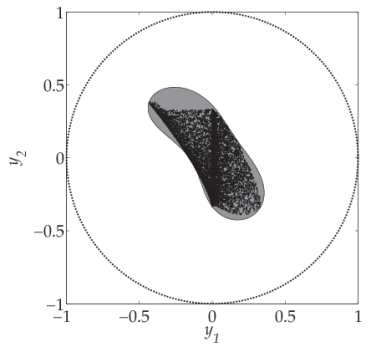

(d) $r=4$

Figure 9: Outer approximations $\mathbf{F}_{r}^{2}$ (light gray) of $\mathbf{F}$ (black dot samples) for Example 4 for $r=1,2,3,4$.

As for Example 1, we report in Table 2 the data related to the semidefinite problems solved by MoseK to compute approximations of increasing degrees, while using Method 1, Method 2 and Method 2 with the lifting strategy. Method 2 fails to compute polynomial approximations of degree higher than eight $(r=4)$, the system running out of memory (indicated with the symbol "-"). The lifting strategy described in Section 5.1.2 overcomes this practical limitation.

Table 2: Comparison of timing results for Example 4

\begin{tabular}{l|c|ccccc}
\hline \multicolumn{2}{c|}{ relaxation order $r$} & 1 & 2 & 3 & 4 & 5 \\
\hline \multirow{3}{*}{ Method 1 } & vars & 66 & 438 & 3137 & 16993 & 73213 \\
& size & 45 & 226 & 1008 & 3387 & 9075 \\
& time (s) & 0.68 & 0.85 & 1.16 & 14.41 & 147.32 \\
\hline \multirow{3}{*}{ Method 2 } & vars & 715 & 12243 & 89695 & - & - \\
& size & 295 & 1957 & 6283 & - & - \\
& time (s) & 0.83 & 3.29 & 52.55 & - & - \\
\multirow{2}{*}{ Method 2 } & vars & 78 & 540 & 3788 & 20216 & 87475 \\
with lifting & size & 51 & 273 & 1262 & 4247 & 11508 \\
& time (s) & 0.68 & 0.96 & 1.83 & 14.44 & 174.80 \\
\hline
\end{tabular}




\section{Discussion and conclusion}

In this work, we propose two methods to approximate polynomial images of basic compact semi-algebraic sets, a numerical approximation alternative to exact computer algebra methods when the latter are too computationally demanding. In its present form, this methodology is applicable to problems of modest size, except if some sparsity can be taken into account, as explained earlier. Therefore, to handle larger size problems, the methodology needs to be adapted. A topic of further investigation is to search for alternative positivity certificates, less demanding than the SOS certificates used in this paper but more efficient than the LP based certificates as defined in Han88, Vas. On the one hand, the latter are appealing since they yield a hierarchy of LP relaxations (as opposed to semidefinite relaxations as in this paper). Moreover, today's LP solvers can handle huge size LP problems, which is far from being the case for semidefinite solvers. On the other hand, it has been shown in [Las09] that generically finite convergence cannot occur for convex problems, except for the linear case. Finally, it could be interesting to look at various compactification procedures to study how the methodology could be generalized to non-compact situations.

\section{Acknowledgments}

This work was partly funded by an award of the Simone and Cino del Duca foundation of Institut de France, a grant of the Gaspard Monge program for optimisation and operations research (PGMO), funded by the Fondation Mathématiques Jacques Hadamard. The authors would like to thank Mohab Safey El Din, Pierre-Loïc Garoche, Jérome Roussel, Alain Sarlette and Xavier Thirioux for fruitful discussions. The authors are also very grateful to the two reviewers for their careful reading, their criticism as well as their detailed feedback.

\section{References}

[AA00] Erling D. Andersen and Knud D. Andersen. The Mosek Interior Point Optimizer for Linear Programming: An Implementation of the Homogeneous Algorithm. In Hans Frenk, Kees Roos, Tamás Terlaky, and Shuzhong Zhang, editors, High Performance Optimization, volume 33 of Applied Optimization, pages 197-232. Springer US, 2000.

[AFP00] Luigi Ambrosio, Nicola Fusco, and Diego Pallara. Functions of bounded variation and free discontinuity problems. Oxford mathematical monographs. Clarendon Press, Oxford, New York, 2000. Autres tirages : 2006.

[Ash72] Robert B. Ash. Real Analysis and Probability. Academic Press, New York, 1972. 
[Bar02] Alexander Barvinok. A Course in Convexity. Graduate studies in mathematics. American Mathematical Society, 2002.

[Bas14] S. Basu. Algorithms in Real Algebraic Geometry: A Survey. ArXiv e-prints, September 2014.

[BPR96] Saugata Basu, Richard Pollack, and Marie-Françoise Roy. On the Combinatorial and Algebraic Complexity of Quantifier Elimination. J. ACM, 43(6):1002-1045, November 1996.

[BPR06] Saugata Basu, Richard Pollack, and Marie-Françoise Roy. Algorithms in Real Algebraic Geometry (Algorithms and Computation in Mathematics). Springer-Verlag New York, Inc., Secaucus, NJ, USA, 2006.

[CK77] B. D. Craven and J. J. Koliha. Generalizations of Farkas' theorem. SIAM J. Math. Anal., 8(6):983-997, 1977.

[Col74] George E. Collins. Quantifier elimination for real closed fields by cylindrical algebraic decomposition-preliminary report. SIGSAM Bull., 8(3):80-90, August 1974.

[GJ88] D. Yu. Grigoriev and N.N. Vorobjov Jr. Solving systems of polynomial inequalities in subexponential time. Journal of Symbolic Computation, $5(1-2): 37-64,1988$.

[Han88] David Handelman. Representing polynomials by positive linear functions on compact convex polyhedra. Pacific Journal of Mathematics, 132(1):35-62, 1988.

[HD12] Hoon Hong and Mohab Safey El Din. Variant quantifier elimination. $J$. Symb. Comput., 47(7):883-901, 2012.

[HLS09] D. Henrion, J. Lasserre, and C. Savorgnan. Approximate volume and integration for basic semialgebraic sets. SIAM Review, 51(4):722-743, 2009.

[Jah10] J. Jahn. Vector Optimization: Theory, Applications, and Extensions. Springer, 2010.

[Las06] Jean B. Lasserre. Convergent sdp-relaxations in polynomial optimization with sparsity. SIAM Journal on Optimization, 17(3):822-843, 2006.

[Las09] J.B. Lasserre. Moments, Positive Polynomials and Their Applications. Imperial College Press optimization series. Imperial College Press, 2009.

[Las10] Jean B. Lasserre. A "joint+marginal" approach to parametric polynomial optimization. SIAM Journal on Optimization, 20(4):1995-2022, 2010.

[Las15] JeanB. Lasserre. Tractable approximations of sets defined with quantifiers. Mathematical Programming, 151(2):507-527, 2015. 
[LN07] Jean B. Lasserre and Tim Netzer. SOS approximations of nonnegative polynomials via simple high degree perturbations. Math. Z., 256(1):99-112, 2007.

[LP10] Jean B. Lasserre and Mihai Putinar. Positivity and optimization for semialgebraic functions. SIAM Journal on Optimization, 20(6):3364-3383, 2010.

[Lue97] David G. Luenberger. Optimization by Vector Space Methods. John Wiley \& Sons, Inc., New York, NY, USA, 1st edition, 1997.

[Lö04] J. Löfberg. Yalmip : A toolbox for modeling and optimization in MATLAB. In Proceedings of the CACSD Conference, Taipei, Taiwan, 2004.

[MHL14] Victor Magron, Didier Henrion, and Jean-Bernard Lasserre. Approximating Pareto curves using semidefinite relaxations. Operations Research Letters, 42(6-7):432 - 437, 2014.

[RF10] H.L. Royden and P. Fitzpatrick. Real Analysis. Featured Titles for Real Analysis Series. Prentice Hall, 2010.

[Tar51] A. Tarski. A Decision Method for Elementary Algebra and Geometry. University of California Press, 1951.

[Trn05] M. Trnovská. Strong duality conditions in semidefinite programming. Journal of Electrical Engineering, 56(12/s):1-5, 2005.

[Vas] F.-H. Vasilescu. Spectral measures and moment problems. Gheondea, Aurelian (ed.) et al., Spectral analysis and its applications. Ion Colojoară anniversary volume. Bucharest: Theta. Theta Series in Advanced Mathematics 2, 173-215 (2003).

[VB94] Lieven Vandenberghe and Stephen Boyd. Semidefinite programming. SIAM Review, 38:49-95, 1994.

[W7̈6] H. R. Wüthrich. Ein Entscheidungsverfahren Für Die Theorie Der ReellAbgeschlossenen Körper. In Komplexität Von Entscheidungsproblemen, Ein Seminar, pages 138-162, London, UK, UK, 1976. Springer-Verlag.

[WCSF01] Benjamin Wilson, David Cappelleri, Timothy W. Simpson, and Mary Frecker. Efficient Pareto Frontier Exploration using Surrogate Approximations. Optimization and Engineering, 2:31-50, 2001.

[WKKM06] Hayato Waki, Sunyoung Kim, Masakazu Kojima, and Masakazu Muramatsu. Sums of squares and semidefinite programming relaxations for polynomial optimization problems with structured sparsity. SIAM Journal on Optimization, 17(1):218-242, 2006. 